\title{
On the Equivalence of the Integral and Differential Bellman Equations in Impulse Control Problems
}

Francois Dufour , Alexey Piunovskiy \& Alexander Plakhov

To cite this article: Francois Dufour, Alexey Piunovskiy \& Alexander Plakhov (2020): On the Equivalence of the Integral and Differential Bellman Equations in Impulse Control Problems, International Journal of Control, DOI: 10.1080/00207179.2020.1786766

To link to this article: https://doi.org/10.1080/00207179.2020.1786766

Accepted author version posted online: 22 Jun 2020.

Submit your article to this journal ¿

山 Article views: 6

Q View related articles $\asymp$

View Crossmark data \lceil 
Publisher: Taylor \& Francis

Journal: International Journal of Control

DOI: $10.1080 / 00207179.2020 .1786766$

\title{
On the Equivalence of the Integral and Differential Bellman Equations in Impulse Control Problems
}

Francois Dufour
Institut Polytechnique de Bordeaux INRIA Bordeaux Sud Ouest, Team. CQFD

IMB Institut de Mathématiques de Bordeaux, Université de Bordeaux, France

dufour@math.u-bordeaux1.fr, Tel: 0033524574165

Alexey Piunovskiy

Department of Mathematical Sciences, University of Liverpool, L69 7ZL, UK. piunov@liv.ac.uk, Tel: 00441517944737

Alexander Plakhov

Center for R\&D in Mathematics and Applications, Department of Mathematics, University of Aveiro, 3810-193, Portugal

and Institute for Information Transmission Problems, Moscow, 127051, Russia plakhov@ua.pt, Tel: 00351234370675

Received 03 Oct 2019, Revised 03 Jun 2020, Accepted 19 Jun 2020

\begin{abstract}
When solving optimal impulse control problems, one can use the dynamic programming approach in two different ways: at each time moment, one can make the decision whether to apply a particular type of impulse, leading to the instantaneous change of the state, or apply no impulses at all; or, otherwise, one can plan an impulse after a certain interval, so that the length of that interval is to be optimized along with the type of that impulse. The first method leads to the differential Bellman equation, while the second method leads to the integral Bellman equation. The target of the current article is to prove the equivalence of those Bellman equations. Firstly, we prove that, for the simple deterministic optimal stopping problem, the equations in the integral and differential form are equivalent under very mild conditions. Here, the impulse means that the uncontrolled process is stopped, i.e., sent to the so called cemetery. After that, the obtained result immediately implies the similar equivalence of the Bellman equations for other models of optimal impulse control. Those include abstract dynamical systems, controlled ordinary differential equations, piece-wise deterministic Markov processes and continuous-time Markov decision processes.
\end{abstract}

Keywords: dynamical system, optimal stopping, impulse control, dynamic programming, total cost.

AMS 2000 subject classification: Primary 49L20; Secondary 49N25, 90C40.

\section{Introduction}

Impulse control of various dynamical systems attracts attention of many researchers: Abakuks (1973), Avrachenkov, Habachi, Piunovskiy and Zhang (2015), Bensoussan and Lions (1984), Costa and Raymundo (2000), de Saporta, Dufour and Geeraert (2017), Dempster and Ye (1995), Dufour and Piunovskiy (2015); Dufour, Horiguchi and Piunovskiy (2016), Hou and Wong (2011), Leander, 
Lenhart and Protopopescu (2015), Menaldi and Robin (2017), Piunovskiy, Plakhov, Torres and Zhang (2019), Piunovskiy, Plakhov and Tumanov (2019), Wei, Yang and Wang (2011), Yushkevich (1989), to mention the most relevant and the most recent works. The underlying system can be described in terms of ordinary (Abakuks, 1973; Avrachenkov et al., 2015; Hou \& Wong, 2011; Leander et al., 2015) or stochastic (Bensoussan \& Lions, 1984; Pescir \& Shiryaev, 2006) differential equations; that was an abstract Markov process in the article by Menaldi and Robin (2017). In the works by Costa and Raymundo (2000), de Saporta et al. (2017), Dempster and Ye (1995) Dufour et al. (2016), Wei et al. (2011), Yushkevich (1989), along with the given deterministic drift, there are spontaneous (or natural) Markov jumps of the state. Such models are called piecewise deterministic Markov processes (PDMP); the drift is usually described by a fixed flow. On the other hand, if there is no drift and the trajectories are piece-wise constant, the mode is called continuous-time Markov decision process (CTMDP) (Dufour \& Piunovskiy, 2015).

By the gradual control we mean that only the local characteristics of the underlying process are under control. In case of PDMP, it means that the rate of the spontaneous/natural jumps, as well as the post-jump distribution are under control. But the impulse control means the following: at particular discrete time moments, the decision maker decides to intervene by instantaneously moving the process to some new point in the state space; that new point can be also random in the cases of CTMDP and PDMP (Dufour \& Piunovskiy, 2015; Dufour et al. 2016; Yushkevich, 1989). Then, restarting at this new point, the process runs until the next intervention and so on. The goal is to minimize the total (expected) accumulated cost which can be discounted (Avrachenkov et al., 2015; Bensoussan \& Lions, 1984; Costa \& Raymundo, 2000; de Saporta et al., 2017; Dempster \& Ye, 1995; Dufour \& Piunovskiy, 2015; Dufour et al., 2016; Menaldi \& Robin, 2017; Piunovskiy et al., 2019a; Wei et al., 2011; Yushkevich, 1989) or not (Abakuks, 1973; Avrachenkov et al., 2015; Bensoussan \& Lions, 1984; Hou \& Wong, 2011; Leander et al., 2015; Piunovskiy et al., 2019a, 2019b; Yushkevich, 1989). The case of long-run average cost was also studied, e.g., in the papers by Avrachenkov et al. (2015) and Yushkevich (1989). Optimal stopping (Abakuks, 1973; Bensoussan \& Lions, 1984; Peskir \& Shiryaev, 2006) is the simplest impulse control: the first one intervention moves the process to the specific state called 'cemetery', which is absorbing and provides no future cost.

Impulse control theory is widely applied to different real-life problems: epidemiology (Abakuks, 1973; Piunovskiy et al., 2019a, 2019b), Internet congestion control (Avrachenkov et al., 2015), reliability (Bensoussan \& Lions, 1984; Dempster \& Ye, 1995), inventory (Bensoussan \& Lions, 1984), economics and finance (Wei et al., 2011), medicine (Bensoussan \& Lions, 1984; Hou \& Wong, 2011), genetics and ecology (Leander et al., 2015), etc.

The most popular method of attack to such problems is dynamic programming (Abakuks, 1973; Avrachenkov et al., 2015; Bensoussan \& Lions, 1984; Costa \& Raymundo, 2000; de Saporta et al., 2017; Dempster \& Ye, 1995; Dufour \& Piunovskiy, 2015; Dufour et al., 2016; Menaldi \& Robin, 2017; Piunovskiy et al., 2019a, 2019b; Wei et al., 2011; Yushkevich, 1989). In Hou and Wong (2011), Leander et al. (2015), versions of the Pontryagin maximum principle were used. When using dynamic programming, two types of the Bellman equation can be studied: integral and differential, the latter one being usually equivalent to what is called (quasi-)variational inequalities (Bensoussan \& Lions, 1984; Costa \& Raymundo, 2000; Wei et al, 2011). Sometimes, it is underlined that these equations are in some sense equivalent (Costa \& Raymundo, 2000). The main goal of the current paper is to prove such equivalence in rather general situations.

The paper is organized in the following way. In Section 2, we consider a very simple optimal stopping problem and prove the equivalence of the integral and differential Bellman equations. Section 3 is devoted to applications of the proved theorem to several special cases: under mild conditions, the integral and differential Bellman equations are equivalent. The very brief conclusion is presented in Section 4. Almost all the statements in the current paper did appear in earlier publications. Our target is to provide a general enough tool for producing one form of the Bellman equation from another. Note that we do not discuss the solvability of the Bellman equations and the existence and properties of optimal control strategies. The question whether a solution to the Bellman equation coincides with the Bellman function is also beyond the scope of this paper. Such information is provided only in passing, when it is possible to give the relevant references.

The following notations are used throughout this paper. $\mathbb{R}_{+}=[0, \infty) ; I\{\cdot\}$ is the indicator 
function; $\delta_{a}(\cdot)$ is the Dirac measure concentrated at the point $a$; $\inf \{\emptyset\} \triangleq \infty ; e^{-\infty} \triangleq 0$; for $a, b \in \mathbb{R}^{d},\langle a, b\rangle$ is the standard scalar product; for a set $D \subset[0, \infty], D-t \triangleq\{s-t, s \in D\}$ for all $t \in[0, \infty)$, and $\infty-t \triangleq \infty$.

\section{Equivalence of the Bellman equations for the optimal stopping problem}

Consider the following optimal stopping problem.

Suppose $\mathbf{Z}$ is an arbitrary state space with the fixed flow $\phi: \mathbf{Z} \times[0, \infty) \rightarrow \mathbf{Z}$ thereon possessing the semigroup property $\phi(x, t+s)=\phi(\phi(x, s), t)$ for all $x \in \mathbf{Z}$ and $(t, s) \in([0, \infty))^{2} ; \phi(x, 0)=x$ for all $x \in \mathbf{Z}$. For each $x \in \mathbf{Z}$, there is a set $\mathbb{T}_{x} \subset[0, \infty]$ such that $\infty \in \mathbb{T}_{x}$ and equation

$$
\mathbb{T}_{\phi(x, t)}=\left(\mathbb{T}_{x}-t\right) \cap[0, \infty]
$$

is satisfied for all $x \in \mathbf{Z}, t \in[0, \infty)$. $\mathbb{T}_{x}$ is the set of all time moments (including $\infty$ ) when the process, starting from $x \in \mathbf{Z}$ and evolving along the flow $\phi$, may be stopped.

Suppose $\overline{\mathbf{X}} \subset \mathbf{Z}$ is a certain subset. For each $x \in \overline{\mathbf{X}}$ the process starting from $x \in \overline{\mathbf{X}}$ must be stopped not later than at the time moment $\bar{t}(x) \in[0, \infty]$. We assume that, for all $x \in \overline{\mathbf{X}}$, $s \in[0, \bar{t}(x)] \cap \mathbb{R}$, the state $\phi(x, s)$ belongs to $\overline{\mathbf{X}}$ and, if $\bar{t}(x)<\infty$, then $\bar{t}(x) \in \mathbb{T}_{x}$. Moreover,

$$
\bar{t}(\phi(x, t))=\bar{t}(x)-t \quad \text { for all } t \in[0, \bar{t}(x)] \cap \mathbb{R} \text {. }
$$

The initial state belongs to $\overline{\mathbf{X}}$, and it is clear that the states from $\mathbf{Z} \times \overline{\mathbf{X}}$ never appear in consideration.

Denote $\mathbf{X}^{\text {stop }} \triangleq\left\{x \in \overline{\mathbf{X}}: \mathbb{T}_{x} \ni 0\right\}$ the subset of the state space at which the process may be stopped. Obviously,

$$
\phi(x, t) \in \mathbf{X}^{\text {stop }} \Longleftrightarrow t \in \mathbb{R} \cap \mathbb{R} \text { for all } x \in \overline{\mathbf{X}}
$$

Finally, we assume that two functions are fixed:

$C: \overline{\mathbf{X}} \rightarrow \mathbb{R}$, the cost rate, satisfying the assumption that, for each $x \in \overline{\mathbf{X}}$, the integral $\int_{0}^{\bar{t}(x)} C(\phi(x, u)) d u$ is well defined and finite. It is sufficient to require that, for each $x \in \overline{\mathbf{X}}$, the function of time $C(\phi(x, t))$ is defined almost everywhere, i.e. $C(\phi(x, \cdot)) \in L^{1}([0, \bar{t}(x)] \cap \mathbb{R})$.

$T: \mathbf{X}^{\text {stop }} \rightarrow \mathbb{R}$, the cost for stopping at the corresponding state $x \in \mathbf{X}^{\text {stop }}$.

The optimal stopping problem consists in calculating, for each $x \in \overline{\mathbf{X}}$, such a time moment $\theta^{*} \in \mathbb{T}_{x} \cap[0, \bar{t}(x)]$ that the total cost

$$
\text { ( } \int_{0}^{\theta^{*}} C(\phi(x, u)) d u+I\left\{\theta^{*}<\infty\right\} T\left(\phi\left(x, \theta^{*}\right)\right)
$$

is minima possible. Clearly, the minimal cost, when starting from the state $x \in \overline{\mathbf{X}}$, that is the Bellman function, equals

$$
V(x)=\inf _{\theta \in \mathbb{T}_{x} \cap[0, \bar{t}(x)]}\left\{\int_{0}^{\theta} C(\phi(x, u)) d u+I\{\theta<\infty\} T(\phi(x, \theta))\right\} .
$$

One can say that at the time moment $\theta$ the decision maker applies the impulse sending the process to the absorbing and costless cemetery state, say, $\Delta \notin \mathbf{Z}$.

Similarly to Piunovskiy et al. (2019a), we accept the following definitions.

- A point $x \in \overline{\mathbf{X}}$ is called singular (in $\overline{\mathbf{X}})$, if equation $x=\phi(\tilde{x}, s)$ has no solutions in $\{(\tilde{x}, s): \tilde{x} \in$ $\overline{\mathbf{X}}, s>0\}$.

- Let $V: \overline{\mathbf{X}} \rightarrow \mathbb{R}$ be a certain function. Then, in case $\bar{t}(x)>0$,

$$
\mathcal{F}_{+}^{V}(x) \triangleq \lim _{t \rightarrow 0+} \frac{1}{t}\left[V(\phi(x, t))-V(x)+\int_{0}^{t} C(\phi(x, u)) d u\right]
$$

provided the limit exists. (If $\bar{t}(x)=0, \mathcal{F}_{+}^{V}(x)$ is undefined.) 
For a nonsingular $x \in \overline{\mathbf{X}}$,

$$
\begin{aligned}
\underline{\mathcal{F}}_{-}^{V}(x) \triangleq & \left\{\varliminf_{t \rightarrow 0+} \frac{1}{t}\left[V(x)-V(\phi(\tilde{x}, s-t))+\int_{-t}^{0} C(\phi(\tilde{x}, s+u)) d u\right]:\right. \\
& (\tilde{x}, s) \in \overline{\mathbf{X}} \times(0, \infty), \phi(\tilde{x}, s)=x\} \subset[-\infty, \infty] .
\end{aligned}
$$

If $x \in \overline{\mathbf{X}}$ is singular, then $\underline{\mathcal{F}}_{-}^{V}(x) \triangleq \emptyset$.

The following theorem is the main theoretical result of the present article.

Theorem 1. Let $V: \overline{\mathbf{X}} \rightarrow \mathbb{R}$ be a certain function, such that $\underline{\lim }_{r \rightarrow \infty} V(\phi(x, r)) \geq 0$ for all $x \in \overline{\mathbf{X}}$ with $\bar{t}(x)=\infty$. Then the following statements are equivalent.

1. The function $V$ satisfies equation

$$
V(x)=\inf _{\theta \in \mathbb{T}_{x} \cap[0, \bar{t}(x)]}\left\{\int_{0}^{\theta} C(\phi(x, u)) d u+I\{\theta<\infty\} T(\phi(x, \theta))\right\}, x \in \overline{\mathbf{X}}
$$

and the following assertions hold true:

- For each $x \in \overline{\mathbf{X}}$, the infimum in Equation (2) is attained on $\Theta(x) \neq \emptyset$, and the set $\Theta(x)$ contains its minimum denoted as $\theta^{*}(x) \in \Theta(x)$.

- The function $V$ is measurable along the flow, that is. $V(\phi(x, t))$ is a measurable function of t for each $x \in \overline{\mathbf{X}}, t \in[0, \bar{t}(x)] \cap \mathbb{R}$.

2. The function $V$ is such that, for each $x \in \overline{\mathbf{X}}$,

$$
\begin{aligned}
& \text { either (a) }\left\{\begin{array}{l}
\mathcal{F}_{+}^{V}(x)=0 \text { and, in case } x \in \mathbf{X}^{\text {stop }}, \\
T(x)-V(x)>0
\end{array}\right. \\
& \text { or, }
\end{aligned}
$$

and, additionally, the following assertions hold true:

(i) - If $\bar{t}(x)>0$ then $\varliminf_{t \rightarrow 0+} V(\phi(x, t)) \geq V(x)$ : the function $V$ is right lower semicontinuous along the flow.

For all $(\tilde{x}, s) \in \overline{\mathbf{X}} \times(0, \infty)$ such that $\phi(\tilde{x}, s)=x, \varlimsup_{t \rightarrow 0+} V(\phi(\tilde{x}, s-t)) \leq V(x)$ : the function $V$ is left upper semicontinuous along the flow. (This assertion is valid by default for singular points $x$.)

(ii) If, for some $s>0$,

$$
\phi(x, t) \notin \mathcal{L} \triangleq\left\{\tilde{x} \in \mathbf{X}^{s t o p}: T(\tilde{x})-V(\tilde{x})=0\right\} \text { for all } 0 \leq t<s,
$$

then

$$
\lim _{t \rightarrow s-} V(\phi(x, t))=V(\phi(x, s)):
$$

the function $V$ is left continuous along the flow.

(iii) - The set

$$
\{t \in[0, \bar{t}(x)] \cap \mathbb{R}: \phi(x, t) \in \mathcal{L}\}
$$

is not empty if $\bar{t}(x)<\infty$. If it is empty (and necessarily $\bar{t}(x)=\infty$ ), then we put $t^{*}(x) \triangleq \infty$. In case it is not empty, it contains its minimum denoted as $t^{*}(x)$.

- In any case, $t^{*}(x) \in \mathbb{T}_{x} \cap[0, \bar{t}(x)]$. 
- For all $t \in\left[0, t^{*}(x)\right]$, equality $t^{*}(\phi(x, t))=t^{*}(x)-t$ is valid and

$$
V(\phi(x, t))-V(x)+\int_{0}^{t} C(\phi(x, u)) d u=0 .
$$

(Hence, $V$ is absolutely continuous along the flow on $\left[0, t^{*}(x)\right]$.)

(iv) If $\bar{t}(x)=\infty$, then $\overline{\lim }_{r \rightarrow \infty} V(\phi(x, r)) \leq 0$, hence $\lim _{r \rightarrow \infty} V(\phi(x, r))=0$.

The functions $\theta^{*}$ and $t^{*}$ in the Statements 1 and 2 above coincide.

Proof. Suppose the Statement 1 is valid and fix $x \in \overline{\mathbf{X}}$ arbitrarily.

Let us prove that the Assertion (i) holds true. Obviously, for any $0<t<\bar{t}(x)$,

$$
V(x) \leq \inf _{\theta \in \mathbb{T}_{x} \cap[t, \bar{t}(x)]}\left\{\int_{0}^{\theta} C(\phi(x, u)) d u+I\{\theta<\infty\} T(\phi(x, \theta))\right\} .
$$

Changing the variables $u-t=v$ and $\theta-t=s$ and using the semigroup property $\phi(x, v+t)=$ $\phi(\phi(x, t), v)$ and Equation (1), we obtain

$$
\begin{aligned}
V(x) \leq & \int_{0}^{t} C(\phi(x, u)) d u \\
& +\inf _{s \in\left(\mathbb{T}_{x}-t\right) \cap[0, \bar{t}(x)-t]=\mathbb{T}_{\phi(x, t)} \cap[0, \bar{t}(\phi(x, t))]}\left\{\int_{0}^{s} C(\phi(\phi(x, t), v) d v+I\{s<\infty\} T(\phi(\phi(x, t), s))\}\right. \\
= & \int_{0}^{t} C(\phi(x, u)) d u+V(\phi(x, t)) .
\end{aligned}
$$

Since $\lim _{t \rightarrow 0+} \int_{0}^{t} C(\phi(x, u)) d u=0$, we have

$$
V(x) \leq \lim _{t \rightarrow 0+} V(\phi(x, t)),
$$

and the first part of the Assertion (i) is established.

Suppose now that $x$ is nonsingular and let $x=\phi(\tilde{x}, s)$ with $s>0$. Similarly to Equation (5), for all $t \in[0, s]$, we have

$V(\phi(\tilde{x}, s-t)) \leq \inf _{\theta \in\left(\mathbb{T}_{x}+t\right) n[t, \tilde{t}(\phi(\tilde{x}(s-t)))]}\left\{\int_{0}^{\theta} C(\phi(\tilde{x}, s-t+u)) d u+I\{\theta<\infty\} T(\phi(\tilde{x}, s-t+\theta))\right\}$.

Changing the variables $u-t=v$ and $\theta-t=\tau$, we get

$V(\phi(\tilde{x}, s-t)\rangle \approx \int_{-t}^{0} C(\phi(\tilde{x}, s+v)) d v$

$$
\begin{aligned}
& +\inf _{\tau \in \mathbb{T}_{x} \cap[0, \bar{t}(\phi(\tilde{x}(s-t)))-t=\bar{t}(x)]}\left\{\int_{0}^{\tau} C(\phi(\tilde{x}, s+v)) d v+I\{\tau<\infty\} T(\phi(\tilde{x}, s+\tau))\right\} \\
& =\int_{-t}^{0} C(\phi(\tilde{x}, s+v)) d v+V(x)
\end{aligned}
$$

because $\phi(\tilde{x}, s)=x$. After taking the upper limit in the obtained inequality, we obtain the second part of the Assertion (i):

$$
\varlimsup_{t \rightarrow 0+} V(\phi(\tilde{x}, s-t)) \leq V(x) .
$$

Now, we are going to prove the main Equation (3) of the Statement 2.

Let $\theta^{*}(x)>0$ and take an arbitrary $t \in\left[0, \theta^{*}(x)\right] \cap \mathbb{R}$. Then Equation (2) remains valid if the infimum is taken over $\theta \in \mathbb{T}_{x} \cap[t, \bar{t}(x)]$. As a consequence, we have the equalities in Equations (5) in (6). By the way, the infimum in Equation (6) is attained at $s \in \Theta(x)-t$, that is, $\Theta(\phi(x, t))=\Theta(x)-t$, and

$$
\theta^{*}(\phi(x, t))=\theta^{*}(x)-t
$$


We showed that

$$
V(x)=\int_{0}^{t} C(\phi(x, u)) d u+V(\phi(x, t)) \text { for } t \in\left[0, \theta^{*}(x)\right]
$$

and the first assertion in the Item (a) of the Statement 2 is proved.

The second assertion follows from the fact that the infimum in Equation (2) is not attained at $\theta=0$ : if $x \in \mathbf{X}^{\text {stop }}$ then $V(x)<T(x)$.

Let $\theta^{*}(x)=0$. Then zero belongs to $\mathbb{T}_{x}$ and provides the infimum in Equation $(2): V(x)=T(x)$. Hence the Assertion (a) fails to hold. To complete the proof of Equation (3), we need to show that $\underline{\mathcal{F}}_{-}^{V} \subset[0, \infty]$.

If $x$ is a singular point, then $\underline{\mathcal{F}}_{-}^{V}=\emptyset$ and the inclusion $\underline{\mathcal{F}}_{-}^{V} \subset[0, \infty]$ is trivially valid,

Suppose $x$ is nonsingular: $x=\phi(\tilde{x}, s)$ with $s>0$. Rewrite Equation (7) as follows:

$$
V(x)-V(\phi(\tilde{x}, s-t))+\int_{-t}^{0} C(\phi(\tilde{x}, s+v)) d v \geq 0 .
$$

Now

$$
\varliminf_{t \rightarrow 0+} \frac{1}{t}\left[V(x)-V(\phi(\tilde{x}, s-t))+\int_{-t}^{0} C(\phi(\tilde{x}, s+v)) d v \geq 0\right.
$$

and the required inclusion $\underline{\mathcal{F}}_{-}^{V} \subset[0, \infty]$ is justified.

Let us check the Assertion (ii).

Suppose $s>0$ and $\phi(x, t) \notin \mathcal{L}$ for all $0 \leq t<s$. In case $\theta^{*}(x)<\infty$, Equations (5) and (6) become equalities if one substitutes $\theta^{*}(x)$ for $t$. Therefore,

$$
V(x)=\int_{0}^{\theta^{*}(x)} C(\phi(x, u)) d u+T\left(\phi\left(x, \theta^{*}(x)\right)\right)=\int_{0}^{\theta^{*}(x)} C(\phi(x, u)) d u+V\left(\phi\left(x, \theta^{*}(x)\right)\right)
$$

and $T\left(\phi\left(x, \theta^{*}(x)\right)\right)=V\left(\phi\left(x, \theta^{*}(x)\right)\right)$, i.e., $\phi\left(x, \theta^{*}(x)\right) \in \mathcal{L}$. Since $\phi(x, t) \notin \mathcal{L}$ for all $0 \leq t<s$, we conclude that $\theta^{*}(x) \geq s>0$. In case $\theta^{*}(x)=\infty$, the inequality $\theta^{*}(x) \geq s>0$ is trivial.

Substituting $s$ for $t$ in Equation (9), we obtain

$$
V(x)=\int_{0}^{s} C(\phi(x, u)) d u+V(\phi(x, s))
$$

After we subtract Equation (9) from this expression, we get

Hence,

$$
0=\int_{t}^{s} C(\phi(x, u)) d u+V(\phi(x, s))-V(\phi(x, t))
$$

$$
\lim _{t \rightarrow s-} V(\phi(x, t))=V(\phi(x, s)) .
$$

The Assertion (ii) is valid.

Let us check the Assertion (iii).

From the proof of the main Equation (3), we see that

if $\theta^{*}(x)>0$, then $x \notin \mathcal{L}$, and

if $\theta^{*}(x)=0$, then $x \in \mathcal{L}$.

By Equation (8), if $0 \leq t<\theta^{*}(x)$, then $\theta^{*}(\phi(x, t))=\theta^{*}(x)-t>0$ and therefore $\phi(x, t) \notin \mathcal{L}$.

In case $t=\theta^{*}(x) \geq 0, \theta^{*}(\phi(x, t))=0$, so that $\phi(x, t) \in \mathcal{L}$. To summarize, if $\theta^{*}(x)=\infty$ (hence $\bar{t}(x)=\infty)$, then the set defined in Equation (4) is empty and $t^{*}(x)=\infty$; and, if $\theta^{*}(x)<\infty$, then $\theta^{*}(x)$ belongs to the set defined in Equation (4) and coincides with its minimum. Therefore, $t^{*}(x)=\theta^{*}(x)$ and $t^{*}(x) \in \mathbb{T}_{x} \cap[0, \bar{t}(x)]$ because $\theta^{*}(x) \in \mathbb{T}_{x} \cap[0, \bar{t}(x)]$. Note, if $\bar{t}(x)<\infty$, then $\theta^{*}(x)<\infty$, and the set defined in Equation (4) is not empty.

The Assertion (iii) is valid: for the last part, see Equations (8) and (9).

For the Assertion (iv), note that

$$
V(x) \leq \lim _{\theta \rightarrow \infty, \theta \in \mathbb{T}_{x}} \int_{0}^{\theta} C(\phi(x, u)) d u=\int_{0}^{\infty} C(\phi(x, u)) d u .
$$


Since $\bar{t}(\phi(x, r))=\infty$ for all $r \geq 0$, and by the semigroup property of the flow $\phi$, we have

$$
V(\phi(x, r)) \leq \int_{0}^{\infty} C(\phi(x, r+u)) d u .
$$

Changing the variables $r+u=v$, we get

$$
V(\phi(x, r)) \leq \int_{r}^{\infty} C(\phi(x, v)) d v .
$$

Therefore,

$$
\varlimsup_{r \rightarrow \infty} V(\phi(x, r)) \leq \lim _{r \rightarrow \infty} \int_{r}^{\infty} C(\phi(x, v)) d v=0 .
$$

The last equality is due to the required property of the function $C$.

The Statement 2 is proved.

Now suppose the Statement 2 is valid.

For an arbitrarily fixed $x \in \overline{\mathbf{X}}$ and $t \in \mathbb{T}_{x} \cap[0, \bar{t}(x)]$, introduce function $\left.h^{t}: \mathcal{} \cdot 0, t\right] \cap \mathbb{R} \rightarrow \mathbb{R}$ by

$$
\left.h^{t}(s)=V(\phi(x, s))-\int_{s}^{t} C(\phi(x, v)) d v-I\{t<\infty\} T(\phi(x), t)\right) .
$$

The case $t=0$ is not excluded; if so, some reasonings below are simplified: e.g., the function $h^{t}$ is clearly a constant. Below, we assume that $t>0$.

First, we show that $h^{t}$ is (monotone) nondecreasing. For $s \in\{0, t)$, consider $\frac{h^{t}(s+\tau)-h^{t}(s)}{\tau}$ under $\tau \in(0, t-s)$. This ratio equals

$$
\frac{V(\phi(x, s+\tau))-V(\phi(x, s))}{\tau}+\frac{1}{\tau} \int_{s}^{s+\tau} C(\phi(x, v)) d v .
$$

Denoting $x^{\prime}=\phi(x, s)$, using the semigroup property of the flow and having in mind that the integral in the right hand side goes to zero as $\tau \rightarrow 0+$, we get

$$
h_{+}^{t^{\prime}}(s)=\lim _{\tau \rightarrow 0}\left\{\frac{V(\phi(x, \tau))-V\left(x^{\prime}\right)}{\tau}+\frac{1}{\tau} \int_{0}^{\tau} C\left(\phi\left(x^{\prime}, u\right)\right) d u\right\}=\mathcal{F}_{+}^{V}(\phi(x, s)),
$$

if $h_{+}^{t}{ }^{\prime}(s)$ and $\mathcal{F}_{+}^{V}(\phi(x, s))$ exist. We emphasize that $\mathcal{F}_{+}^{V}(\phi(x, s))$ and $h_{+}^{t}{ }^{\prime}(s)$ exist (or do not exist) simultaneously.

In the similar way one calculates the lower left derivative of $h^{t}$ and concludes that

$$
{\underline{h_{-}^{t}}}^{\prime}(s) \in \underline{\mathcal{F}}_{-}^{V}(\phi(x, s))
$$

for all $s \in(0, t] \cap \mathbb{R}$. Note that, for each $s>0$, the point $\phi(x, s)$ is nonsingular.

According to the Statement 2, for $x \in \mathcal{L}$ we have $\underline{\mathcal{F}}_{-}^{V}(x) \subset[0, \infty)$ and, otherwise, $\mathcal{F}_{+}^{V}(x)=0$. By Equations (10) and (11) we conclude that, if $\phi(x, s) \notin \mathcal{L}$, then $h_{+}^{t}{ }^{\prime}(s)$ exists and equals zero, and, if $\phi(x, s) \in \mathcal{L}$ and $s>0$, then $h_{-}^{t}(s) \geq 0$. Taking into account the Assertion (i), we conclude that the function $h^{t}$ is also right lower semicontinuous on $[0, t)$ and left upper semicontinuous on (0, $t] \cap \mathbb{R}$. Therefore $h^{t}(\cdot)$ satisfies all the conditions of Lemma 1 (see Appendix) and hence is nondecreasing. Thus, the function $h^{\bar{t}(x)}(\cdot)$ is measurable, and the last assertion of the Statement 1 is valid: the function $\int_{s}^{\widetilde{t}(x)} C(\phi(x, v)) d v$ is obviously measurable.

If $t<\infty$, we have

$$
h^{t}(t)=V(\phi(x, t))-T(\phi(x, t)),
$$

and, by virtue of Equation (3), $h^{t}(t) \leq 0$ because the difference $V(y)-T(y)$ can never be strictly positive. Thus,

$$
h^{t}(0)=V(x)-\int_{0}^{t} C(\phi(x, v)) d v-T(\phi(x, t)) \leq h^{t}(t) \leq 0 .
$$


If, otherwise, $t=\infty$, then $\bar{t}(x)=\infty$, and we have for all $s \in[0, \infty)$

$$
h^{t}(0) \leq h^{t}(s)=V(\phi(x, s))-\int_{s}^{\infty} C(\phi(x, v)) d v .
$$

According to the key property of the function $C$, the integral in the right hand side of this relation is finite and goes to 0 as $s \rightarrow \infty$. If $h^{t}(u) \geq \varepsilon>0$ for some $u \geq 0, \varepsilon>0$ then, for all $s \geq u$, $h^{t}(s) \geq h^{t}(u) \geq \varepsilon$ and

$$
V(\phi(x, s)) \geq \varepsilon+\int_{s}^{\infty} C(\phi(x, v)) d v,
$$

i.e., $V(\phi(x, r)) \geq \frac{\varepsilon}{2}$ for all big enough $r$ which contradicts the Assertion (iv). We conelude that $h^{t}(s) \leq 0$ for all $s \in[0, \infty)$ and, in particular,

$$
h^{t}(0)=V(x)-\int_{0}^{\infty} C(\phi(x, u)) d u \leq 0 .
$$

Equations (13) and (14) imply that, for all $x \in \overline{\mathbf{X}}$,

$$
V(x) \leq \inf _{t \in \mathbb{T}_{x} \cap[0, \bar{t}(x)]}\left\{\int_{0}^{t} C(\phi(x, u)) d u+I\{t<\infty\} T(\phi(x, t))\right\} .
$$

(If $t=0 \in \mathbb{T}_{x}$, then inequality $V(x) \leq T(x)$ follows directly from Equation (3).)

Now we intend to show that, for each $x \in \overline{\mathbf{X}}$, for $t^{*}(x)$, the function $h^{t^{*}(x)}(\cdot)$ is identical zero for all $s \in\left[0, t^{*}(x)\right] \cap \mathbb{R}$. If $t^{*}(x)=0$ then $h^{t^{*}(x)}\left(t^{*}(x)\right)=0$. Below, we assume that $t^{*}(x)>0$. Note also that $t^{*}(x) \in \mathbb{T}_{x} \cap[0, \bar{t}(x)]$ according to the Assertion (iii).

For $0 \leq s<t^{*}(x)$ we have $\phi(x, s) \notin \mathcal{L}$ and therefore $h^{t^{*}(x)^{\prime}}{ }_{+}^{\prime}(s)=0$. According to the Assertion (ii), the function $s \mapsto V(\phi(x, s))$, and therefore also the function $h^{t^{*}(x)}(\cdot)$, are left continuous for finite $0<s \leq t^{*}(x)$. Hence, by Lemma 2 (see Appendix), $h^{t^{*}(x)}(\cdot)$ is constant on $\left[0, t^{*}(x)\right] \cap \mathbb{R}$.

Let $t^{*}(x)$ be finite. The Assertion (iii) states that $\phi\left(x, t^{*}(x)\right) \in \mathcal{L}$, and therefore,

$$
h^{t^{*}(x)}\left(t^{*}(x)\right)=K\left(\phi\left(x, t^{*}(x)\right)\right)-T\left(\phi\left(x, t^{*}(x)\right)\right)=0 .
$$

It follows that $h^{t^{*}(x)}(s)=0$ for all $0 \leq s \leq t^{*}(x)$, and, in particular,

$$
h(0)=V(x)-\int_{0}^{t^{*}(x)} C(\phi(x, u)) d u-T\left(\phi\left(x, t^{*}(x)\right)\right)=0 .
$$

If, otherwise, $t^{*}(x)=\infty$ (hence, $\left.\bar{t}(x)=\infty\right)$, then the constant function $h^{t^{*}(x)}(s)$, by the Assertion (iv) and by the key property of the function $C$, is the sum of two functions going to 0 as $s \rightarrow \infty$. Therefore it is the null function, and again

$$
h(0)=V(x)-\int_{0}^{\infty} C(\phi(x, u)) d u=0 .
$$

This proves that

$$
\begin{aligned}
V(x) & =\int_{0}^{t^{*}(x)} C(\phi(x, u)) d u+I\left\{t^{*}(x)<\infty\right\} T\left(\phi\left(x, t^{*}(x)\right)\right) \\
& \geq \inf _{t \in \mathbb{T}_{x} \cap[0, \bar{t}(x)]}\left\{\int_{0}^{t} C(\phi(x, u)) d u+I\{t<\infty\} T(\phi(x, t))\right\} .
\end{aligned}
$$

As a consequence of Equations (15) and (16) we obtain that Equation (2) is valid and $t^{*}(x) \in$ $\Theta(x) \neq \emptyset$.

It remains to show that $t^{*}(x)$ equals the minimum of the set $\Theta(x)$. Without loss of generality, we assume that $t^{*}(x)>0$.

Fix an arbitrary $0 \leq t<t^{*}(x)$ in the definition of $h^{t}$, such that $t \in \mathbb{T}_{x} \cap[0, \bar{t}(x)]$. Since $\phi(x, t) \notin \mathcal{L}$, by Equation (12) we have

$$
h^{t}(t)=V(\phi(x, t))-T(\phi(x, t))<0:
$$


recall that the difference $V(y)-T(y)$ can never be strictly positive. Since the function $h^{t}(\cdot)$ is nondecreasing, we conclude that

$$
h^{t}(0)=V(x)-\int_{0}^{t} C(\phi(x, u)) d u-T(\phi(x, t)) \leq h^{t}(t)<0 .
$$

As a result we have

$$
V(x)<\int_{0}^{t} C(\phi(x, u)) d u+T(\phi(x, t))
$$

that is, $t \notin \Theta(x)$ for $0 \leq t<t^{*}(x)$. This implies that $t^{*}(x)=\inf \Theta(x)$. The Statement 1 is valid.

Remark 1. (a) The first half of the proof (the Statement $1 \Longrightarrow$ the Statement 2) does not use the last assertion about the measurability of the function $V$. Therefore, in the framework of Theorem 1, if all the other assertions of the Statement 1 are valid, then $V(\phi(x, t))$ is a measurable function of $t$ for each $x \in \overline{\mathbf{X}}, t \in[0, \bar{t}(x)] \cap \mathbb{R}$.

(b) The second half of the proof (the Statement $2 \Longrightarrow$ the Statement 1 ) does not use the last part of the Assertion (iii). Therefore, in the framework of Theorem 1 if all the other assertions of the Statement 2 are valid, then

- For all $t \in\left[0, t^{*}(x)\right]$, equality $t^{*}(\phi(x, t))=t^{*}(x)-t$ is valid and

$$
V(\phi(x, t))-V(x)+\int_{0}^{t} C(\phi(x, u)) d u=0
$$

automatically.

Condition 1. For each point $x \in \overline{\mathbf{X}}$, if $x=\phi\left(\tilde{x}_{1}, s_{1}\right)=\phi\left(\tilde{x}_{2}, s_{2}\right)$ with $\tilde{x}_{1}, \tilde{x}_{2} \in \overline{\mathbf{X}}$, then either $\tilde{x}_{1}=\phi\left(\tilde{x}_{2}, s_{2}-s_{1}\right)$, or $\tilde{x}_{2}=\phi\left(\tilde{x}_{1}, s_{1}-s_{2}\right)$.

Under Condition 1, one can define the flow in the reverse time for each nonsingular point $x \in \overline{\mathbf{X}}$.

- If there is a pair $\left(x_{\text {sing }}, S_{x}>0\right)$ such that $x_{\text {sing }}$ is singular and $x=\phi\left(x_{\text {sing }}, S_{x}\right)$, then, for all $t \leq S_{x}$, we put

$$
\phi(x,-t) \triangleq \phi\left(x_{\text {sing }}, S_{x}-t\right)
$$

Note, the pair $\left(x_{\text {sing }}, S_{x}\right)$ is unique.

- If there are no singular points $x_{\text {sing }}$ such that $x=\phi\left(x_{\text {sing }}, s\right)$ with $s>0$, then we put

$$
S_{x} \triangleq \sup \{s: \exists(\tilde{x}, s): x=\phi(\tilde{x}, s\}>0
$$

and, for all $t<S_{x}$,

$$
\phi(x,-t) \triangleq \phi(\tilde{x}, s-t)
$$

where $(\tilde{x}, s)$ is such a pair that $s>t$ and $x=\phi(\tilde{x}, s)$.

Now, for each nonsingular $x \in \overline{\mathbf{X}}$, there is a number $S_{x}>0$ such that the flow $\phi$ is well defined for all $t \geq-S_{x}$ or $t>-S_{x}$ and, like initially, possesses the semigroup property. Note that, for each nonsingular point $x \in \overline{\mathbf{X}}, \underline{\mathcal{F}}_{-}^{V}(x)$ is a singleton equal to

$$
\begin{aligned}
& \varliminf_{t \rightarrow 0+} \frac{1}{t}\left[V(x)-V(\phi(x,-t))+\int_{-t}^{0} C(\phi(x, v)) d v\right] \\
= & \varliminf_{t \rightarrow 0-} \frac{1}{t}\left[V(\phi(x, u))-V(x)+\int_{0}^{t} C(\phi(x, v)) d v\right] .
\end{aligned}
$$

Condition 2. Condition 1 is satisfied; for each singular $x \in \overline{\mathbf{X}}$, the function of time $C(\phi(x, t))$, $t \geq 0$ is continuous, and, for each nonsingular $x \in \overline{\mathbf{X}}$, the function of time $C(\phi(x, t)), t>-S_{x}$ is continuous. 
For example, Condition 2 is satisfied if Condition 1 is valid, $\overline{\mathbf{X}}$ is a topological space, the flow $\phi$ is continuous and the function $C$ is continuous.

If Condition 2 is satisfied, then one can present several assertions of the Statement 2 in Theorem 1 in a clearer way.

- In the Assertion (a),

$$
\mathcal{F}_{+}^{V}(x)=V_{+}^{\prime}(x)+C(x)=0
$$

where

$$
V_{+}^{\prime}(x) \triangleq \lim _{t \rightarrow 0+} \frac{1}{t}[V(\phi(x, t))-V(x)]
$$

is the right derivative of $V$ along the flow, and this limit exists.

- In the Assertion (b), either $x$ is singular, or

$$
\underline{V}_{-}^{\prime}(x)+C(x) \geq 0
$$

where

$$
\underline{V}_{-}^{\prime}(x) \triangleq \varliminf_{t \rightarrow 0+} \frac{1}{t}[V(x)-V(\phi(x,-t))]=\varliminf_{u \rightarrow 0-} \frac{1}{u}[V(\phi(x, u))-V(x)]
$$

is the lower left derivative of $V$ along the flow.

- The second part of the Assertion (i) can be reformulated as follows:

- In case $x$ is nonsingular,

$$
\varlimsup_{t \rightarrow 0-} V(\phi(x, t)) \leq V(x):
$$

the function $V$ is left upper semicontinuous along the flow.

Finally, in case Condition 2 is satisfied and the function $V$ is differentiable along the flow, after we introduce its derivative

$$
V^{\prime}(x) \triangleq \lim _{t \rightarrow 0} \frac{1}{t}[V(\phi(x, t))-V(x)]
$$

(the right derivative if $x$ is singular, and the left derivative if $\bar{t}(x)=0$ ), then Equation (3) takes the following form:

$$
\begin{aligned}
& V^{\prime}(x)+C(x) \geq 0, T(x)-V(x) \geq 0, \quad\left(V^{\prime}(x)+C(x)\right)(T(x)-V(x))=0 \\
\Longleftrightarrow & \min \left\{V^{\prime}(x)+C(x), \quad T(x)-V(x)\right\}=0 .
\end{aligned}
$$

If $x$ is singular and $\bar{t}(x)=0$ then $V^{\prime}(x)$ is undefined and $T(x)-V(x)=0$.

Similar relations in the impulse control theory are sometimes called (quasi-)variational inequalities (Bensoussan \& Lions, 1984; Costa \& Raymundo, 2000; Wei et al., 2011).

\section{Bellman equations in the optimal impulse control problems}

In this section, we illustrate how Theorem 1 can be used when studying different particular models.

\subsection{Impulse control of dynamical systems}

A version of Theorem 1 about the equivalence of the integral and differential forms of the Bellman equation, under a little more restrictive conditions, was proved in Piunoskiy et al. (2019a) deliberately for the problem of optimal impulse control of deterministic dynamical systems. Below, we show how to adjust Theorem 1 for this case.

The model is defined by the following statements.

- $\mathbf{X}$ is the state space, a Borel subset of a complete separable metric space with metric $\rho_{X}$ and the Borel $\sigma$-algebra. 
- $\phi(\cdot, \cdot): \mathbf{X} \times[0, \infty) \rightarrow \mathbf{X}$ is the flow possessing the semigroup property $\phi(x, t+s)=$ $\phi(\phi(x, s), t)$ for all $x \in \mathbf{X}$ and $(t, s) \in([0, \infty))^{2} ; \phi(x, 0)=x$ for all $x \in \mathbf{X}$. Between the impulses, the state changes according to the flow.

- $\mathbf{A}$ is the action space, again a Borel subset of a complete separable metric space with metric $\rho_{A}$ and the Borel $\sigma$-algebra.

- $l(\cdot, \cdot): \mathbf{X} \times \mathbf{A} \rightarrow \mathbf{X}$ is the mapping describing the new state after the corresponding action/impulse is applied.

- $C^{g}(x)$ is the (gradual) cost rate.

- $C^{i}(x, a)$ is the cost associated with the actions/impulses applied at the corresponding states.

The dynamic programming approach, developed in Piunovskiy et al. (2019a) for the case of total undiscounted cost, leads to the following Bellman equation in the integral form:

$V(x)=\inf _{\theta \in[0, \infty]}\left\{\int_{0}^{\theta} C^{g}(\phi(x, u)) d u+I\{\theta<\infty\} \inf _{a \in \mathbf{A}}\left\{C^{i}(\phi(x, \theta), a)+V(l(\phi(x, \theta), a))\right\}\right\}, \quad x \in \mathbf{X}$.

We leave aside the conditions when this equation has a solution coincident with the Bellman function (the minimal total cost when starting from the state $x \in \mathbf{X}$ ) and also the properties of the function $V$ and of the infimum with respect to $a$ in Equation (18). Assume only that the integral $\int_{0}^{\infty} C^{g}(\phi(x, u)) d u$ is well defined and finite, a solution $V$ to Equation (18) exists, and $\underline{\lim }_{r \rightarrow \infty} V(\phi(x, r)) \geq 0$ for all $x \in \mathbf{X}$. The interested reader can find the corresponding conditions, when these assumptions are valid, in Piunovskiy et al. (2019a).

Suppose a function $V$ is a solution to Equation (18), the infimum with respect to $\theta$ is attained on $\Theta(x) \neq \emptyset$, and the set $\Theta(x)$ contains its minimum. Let us denote

$$
\begin{gathered}
\mathbf{Z}=\overline{\mathbf{X}}=\mathbf{X} ; \mathbb{T}_{x} \triangleq[0, \infty] ; \mathbf{X}^{s t o p}=\mathbf{X} ; t(x) \equiv \infty ; \mathbf{X}^{s t o p} \triangleq \mathbf{X} ; C(x) \triangleq C^{g}(x) ; \\
T(x) \triangleq \inf _{a \in \mathbf{A}}\left\{C^{i}(x, a)+V(l(x, a))\right\}, x \in \mathbf{X} .
\end{gathered}
$$

Now the function $V$ is a solution to Equation (2) and hence the Statement 2 of Theorem 1 is valid. We substitute the expression for the function $T$ and conclude that, for each $x \in \mathbf{X}$,

$$
\begin{aligned}
& \text { either (a) }\left\{\begin{array}{l}
\mathcal{F}_{+}^{V}(x)=0 \text { and } \\
\inf _{a \in \mathbf{A}}\left\{C^{i}(x, a)+V(l(x, a))-V(x)\right\}>0
\end{array}\right. \\
& \text { or, if the Assertion (a) fails to hold, then }
\end{aligned}
$$

$$
\text { (b) }\left\{\begin{array}{l}
\mathcal{F}_{-}^{V}(x) \subset[0, \infty] \text { and } \\
\inf _{a \in \mathbf{A}}\left\{C^{i}(x, a)+V(l(x, a))-V(x)\right\}=0 .
\end{array}\right.
$$

$\mathcal{F}_{+}^{V}(x)$ and $\mathcal{F}_{-}(x)$ are as defined in Section 2 with $C$ being replaced with $C^{g}$.

Equation (20) represents the Bellman equation in the differential form; these relations can be also called quasi-variational inequalities. The Assertions (i)-(iv) are also valid. As explained at the end of Section 2, under mild conditions, Equation (20) takes clearer form.

In the inverse direction, suppose a function $V$ satisfies the differential Bellman Equation (20), along with the Assertions (i)-(iv) of the Statement 2 of Theorem 1, where $\mathbf{X}^{\text {stop }}, C(\cdot)$, and $T(\cdot)$ are replaced in accordance with Equation (19). Then, by Theorem 1, function $V$ satisfies Equation (2) and, after we substitute the expressions given in Equation (19) for $C(\cdot)$ and $T(\cdot)$, we conclude that the function $V$ satisfies the integral Bellman Equation (18), the infimum is attained on $\Theta(x) \notin \emptyset$, and the set $\Theta(x)$ contains its minimum.

Example. Consider the standard Susceptible-Infected-Recovered (SIR) epidemic described by differential equations

$$
\begin{aligned}
& \dot{x}(t)=-\beta x(t) y(t), \\
& \dot{y}(t)=\beta x(t) y(t)-\gamma y(t),
\end{aligned}
$$


where $x$ is the volume of the susceptible population, $y$ is the amount of infectives, $\gamma>0$ is the recover rate, and $\beta>0$ is the rate of the infection transmission. At any moment, one can isolate all the infectives: $l(x, y)=(x, 0)$. Here $\mathbf{X}=\mathbb{R}_{+}^{2}$ and $\mathbf{A}=\{$ isolate all $\}$ is a singleton and, hence, the argument $a$, as in Equations (19) and (20), will be omitted below. The flow is defined by the differential Equations (21). The process is terminated as soon as the first impulse is applied, i.e., we deal with the optimal stopping problem. The objective is to minimize the total number of new infectives plus the total cost of isolation: $C^{g}(x, y)=\beta x y ; C^{i}(x, y)=c y$, where $c>0$ is the cost of isolation of one infective.

The integral Bellman Equation (18) takes the intuitively obvious form:

$$
V\left(x_{0}, y_{0}\right)=\inf _{\theta \in[0, \infty]}\left\{\int_{0}^{\theta} \beta x(u) y(u) d u+I\{\theta<\infty\}[c y(\theta)+V(x(\theta), 0)]\right\} .
$$

Here and below, $\left(x_{0}, y_{0}\right) \in \mathbb{R}_{+}^{2}$ is the initial condition for the system defined in Equation (21).

Since Condition 2 is satisfied, the differential Bellman Equation (20) takes the following form in all the areas where the function $V$ is differentiable:

$$
\begin{aligned}
& \min \left\{\quad \frac{\partial V}{\partial x}[-\beta x y]+\frac{\partial V}{\partial y}[\beta x y-\gamma y]+\beta x y ;\right. \\
& c y+V(x, 0)-V(x, y)\}=0 \text {. }
\end{aligned}
$$

One can recognise here the (quasi-)variational inequalities.

Clearly, if $y_{0}=0$, then, for each $x_{0} \in \mathbb{R}_{+}, x(r)=x_{0}$ and $y(r)=0$ for all $r \in \mathbb{R}_{+}$. Hence, in this case, according to the condition in Theorem $1, \lim _{r} \rightarrow_{\infty} V(x(r), y(r))=V\left(x_{0}, 0\right) \geq 0$. Equality $V\left(x_{0}, 0\right)=0$, for all $x_{0} \in \mathbb{R}_{+}$, follows directly from the integral equation: the infimum is attained at $\theta=\infty$. The same equality $V\left(x_{0}, 0\right)=0$ follows also from the Assertion (iv) of Theorem 1: for each $x_{0} \in \mathbb{R}_{+}, \lim _{r \rightarrow \infty} V(x(r), 0)=V\left(x_{0}, 0\right)=0$.

Although the explicit form of the function $V$ is under question, the shape of the area

$$
\{(x, y): V(x, y)=c y\}
$$

where the impulse is needed, is known (Abakuks, 1973). Other modifications of the presented example were investigated in Piunovskiy et al. (2019a, 2019b).

\subsection{Impulse control of piece-wise deterministic Markov processes}

Consider the model investigated in Dufour et al. (2016), which is defined by the following statements.

- $\mathbf{Y}$ is the state space, assumed to be an open subset of $\mathbb{R}^{d}$ and $\partial \mathbf{Y}$ denotes the boundary of $\mathrm{Y}$.

- $\bar{\phi}(y, t): \mathbb{R}^{d} \times[0, \infty) \rightarrow \mathbb{R}^{d}$ is the fixed flow.

- $\mathbf{\Xi}=\{y \in \partial \mathbf{Y}: y=\bar{\phi}(\tilde{y}, t)$ for some $\tilde{y} \in \mathbf{Y}$ and $t \in(0, \infty)\}$ is the active boundary and $\overline{\mathbf{Y}} \triangleq \mathbf{Y} \cup \boldsymbol{\Xi}$. For $y \in \overline{\mathbf{Y}}$ the notation $\bar{t}(y)=\inf \{t \in[0, \infty): \bar{\phi}(y, t) \in \boldsymbol{\Xi}\}$ is in use: starting from the point $y \in \overline{\mathbf{Y}}$, an impulse must be applied not later than at the time moment $\bar{t}(y)$.

- $\mathbf{A}$ is the action space, assumed to be a Borel space. $\mathbf{A}^{i} \in \mathcal{B}(\mathbf{A})\left(\right.$ respectively $\mathbf{A}^{g} \in \mathcal{B}(\mathbf{A})$ ) is the set of impulsive (respectively gradual) actions satisfying $\mathbf{A}=\mathbf{A}^{i} \cup \mathbf{A}^{g}$ with $\mathbf{A}^{i} \cap \mathbf{A}^{g}=\emptyset$.

- The set of admissible actions in the state $y \in \overline{\mathbf{Y}}$ is $\mathbf{A}(y) \in \mathcal{B}(\mathbf{A})$. For all $y \in \mathbf{\Xi}, \mathbf{A}(y) \cap \mathbf{A}^{i} \neq \emptyset$, and $\mathbf{A}(y) \cap \mathbf{A}^{g} \neq \emptyset$ for all $y \in \overline{\mathbf{Y}}$. Below, $\mathbf{A}^{i}(y) \triangleq \mathbf{A}(y) \cap \mathbf{A}^{i}$ and $\mathbf{A}^{g}(y) \triangleq \mathbf{A}(y) \cap \mathbf{A}^{g}$ are admissible impulsive and gradual actions, correspondingly, in the state $y \in \overline{\mathbf{Y}}$.

$$
\mathbf{K}^{i}=\left\{(y, a) \in \overline{\mathbf{Y}} \times \mathbf{A}: a \in \mathbf{A}^{i}(y)\right\} \in \mathcal{B}\left(\mathbf{Y}^{\text {stop }} \times \mathbf{A}^{i}\right)
$$

where $\mathbf{Y}^{\text {stop }}=\left\{y \in \overline{\mathbf{Y}}: \mathbf{A}^{i}(y) \neq \emptyset\right\} \in \mathcal{B}(\overline{\mathbf{Y}})$ is the set of states where impulses are allowed.

$$
\mathbf{K}^{g}=\left\{(y, a) \in \overline{\mathbf{Y}} \times \mathbf{A}: a \in \mathbf{A}^{g}(y)\right\} \in \mathcal{B}\left(\overline{\mathbf{Y}} \times \mathbf{A}^{g}\right),
$$


It is assumed that $\mathbf{K}^{i}$ (respectively, $\mathbf{K}^{g}$ ) contains the graph of a measurable function from $\mathbf{Y}^{\text {stop }}$ to $\mathbf{A}^{i}$ (respectively, from $\overline{\mathbf{Y}}$ to $\mathbf{A}^{g}$ ).

- The controlled jump intensity $\lambda$ which is a bounded $[0, \infty)$-valued measurable function defined on $\mathbf{K}^{g}$.

- The stochastic kernel $Q$ on $\mathbf{Y}$ given $\mathbf{K} \triangleq \mathbf{K}^{i} \cup \mathbf{K}^{g}$ satisfying $Q(\mathbf{Y} \backslash\{y\} \mid y, a)=1$ for any $(y, a) \in \mathbf{K}^{g}$. It describes the state of the process after anyone jump. Below, for $(y, a) \in \mathbf{K}^{g}$,

$$
q(d \tilde{y} \mid y, a)=\lambda(y, a)\left[Q(d \tilde{y} \mid y, a)-\delta_{y}(d \tilde{y})\right]
$$

is the controlled infinitesimal generator of the unforced (natural) jumps.

- $C^{g}\left(y, a^{g}\right)$ is the (gradual) cost rate, defined for all $\left(y, a^{g}\right) \in \mathbf{K}^{g}$.

- $C^{i}\left(y, a^{i}\right)$ is the cost associated with the impulsive actions, defined for all $\left(y, a^{i}\right) \in \mathbf{K}^{i}$.

Below,

$$
\mathbb{T}_{y} \triangleq\left\{t: \quad \bar{\phi}(y, t) \in \mathbf{Y}^{s t o p}\right\} \cup\{\infty\}
$$

is the set of time moments, after a jump of the process into the state $y$, when the decision maker may apply an impulsive action.

The dynamic programming approach, developed in Dufour et al. (2016), for the case of total discounted cost with the discount factor $\alpha>0$, leads to the following Bellman equation in the integral form:

$$
\begin{aligned}
W(y)= & \inf _{\theta \in \mathbb{T}_{y} \cap[0, \bar{t}(y)]}\left\{\int _ { 0 } ^ { \theta } e ^ { - \alpha u } \operatorname { i n f } _ { a ^ { g } \in \mathbf { A } ^ { g } ( \overline { \phi } ( y , u ) ) } \left\{C^{g\left(\bar{\phi}(y, u), a^{g}\right)}\right.\right. \\
& \left.+\lambda\left(\bar{\phi}(y, u), a^{g}\right)\left[\int_{\mathbf{Y}} W(\tilde{y}) Q\left(d \tilde{y} \mid \bar{\phi}(y, u), a^{g}\right)-W(\bar{\phi}(y, u))\right]\right\} d u \\
& \left.+e^{-\alpha \theta} \inf _{a^{i} \in \mathbf{A}^{i}(\bar{\phi}(y, \theta))}\left\{C^{i}\left(\bar{\phi}(y, \theta), a^{i}\right)+\int_{\mathbf{Y}} W(\tilde{y}) Q\left(d \tilde{y} \mid \bar{\phi}(y, \theta), a^{i}\right)\right\}\right\}, \quad y \in \overline{\mathbf{Y}} .
\end{aligned}
$$

We leave aside the conditions when this equation has a solution coincident with the Bellman function (the minimal discounted cost when starting from the state $y \in \overline{\mathbf{Y}}$ ) and also the properties of the function $W$ and of the infima with respect to $a^{g}$ and $a^{i}$ in Equation (22). Assume only that a solution $W$ to Equation (22) exists, the functions $C^{g}\left(y, a^{g}\right)$ and $W(y)$ are bounded, and the function

$$
\inf _{a^{g} \in \mathbf{A}^{g}(y)}\left\{C^{g}\left(y, a^{g}\right)+\lambda\left(y, a^{g}\right)\left[\int_{\mathbf{Y}} W(\tilde{y}) Q\left(d \tilde{y} \mid y, a^{g}\right)-W(y)\right]\right\}
$$

is measurable along the flow $\bar{\phi}$ in $\overline{\mathbf{Y}}$. The interested reader can find the corresponding conditions, when these assumptions are valid, in Dufour et al. (2016).

In order to apply Theorem 1, we need some additional constructions. We extend the state space and introduce $\mathbf{Z} \triangleq \mathbb{R}^{d+1}$ and $\overline{\mathbf{X}} \triangleq \overline{\mathbf{Y}} \times \mathbb{R} \subset \mathbf{Z}$; the generic elements of $\mathbf{Z}$ and $\overline{\mathbf{X}}$ are denoted as $x=(y, \tau)$, the additional component $\tau$ representing time. The flow $\phi$ in $\mathbf{Z}$, the set $\mathbf{X}^{\text {stop }}$, the sets $\mathbb{T}_{x}$ and function $\bar{t}(x)$ for $x=(y, \tau)$ are introduced in the natural way:

$$
\phi(x, t) \triangleq(\bar{\phi}(y, t), \tau+t) ; \mathbf{X}^{\text {stop }} \triangleq \mathbf{Y}^{\text {stop }} \times \mathbb{R} ; \mathbb{T}_{x} \triangleq \mathbb{T}_{y} \times \mathbb{R} ; \bar{t}(x) \triangleq \bar{t}(y) .
$$

Suppose $W$ is a bounded function on $\overline{\mathbf{Y}}$ and introduce function $V$ on $\overline{\mathbf{X}}$ by

$$
V(x) \triangleq e^{-\alpha \tau} W(y) \quad \text { for } x=(y, \tau) .
$$


Now the function $W$ satisfies Equation (22) if and only if the function $V$ satisfies equation

$$
\begin{aligned}
V(x)= & \inf _{\theta \in \mathbb{T}_{y} \cap[0, \bar{t}(y)]}\left\{\int _ { 0 } ^ { \theta } e ^ { - \alpha ( \tau + u ) } \operatorname { i n f } _ { a ^ { g } \in \mathbf { A } ^ { g } ( \overline { \phi } ( y , u ) ) } \left\{C^{g}\left(\bar{\phi}(y, u), a^{g}\right)\right.\right. \\
& \left.+\lambda\left(\bar{\phi}(y, u), a^{g}\right)\left[\int_{\mathbf{Y}} W(\tilde{y}) Q\left(d \tilde{y} \mid \bar{\phi}(y, u), a^{g}\right)-W(\bar{\phi}(y, u))\right]\right\} d u \\
& \left.+e^{-\alpha(\tau+\theta)} \inf _{a^{i} \in \mathbf{A}^{i}(\bar{\phi}(y, \theta))}\left\{C^{i}\left(\bar{\phi}(y, \theta), a^{i}\right)+\int_{\mathbf{Y}} W(\tilde{y}) Q\left(d \tilde{y} \mid \bar{\phi}(y, \theta), a^{i}\right)\right\}\right\} \\
= & \inf _{\theta \in \mathbb{T}_{x} \cap[0, \bar{t}(x)]}\left\{\int_{0}^{\theta} C(\phi(x, u)) d u+I\{\theta<\infty\} T(\phi(x, \theta))\right\}, \quad x \in \overline{\mathbf{X}},
\end{aligned}
$$

where, for $x=(y, \tau)$,

$$
\begin{aligned}
& C(x) \triangleq e^{-\alpha \tau} \inf _{a^{g} \in \mathbf{A}^{g}(y)}\left\{C^{g}\left(y, a^{g}\right)+\lambda\left(y, a^{g}\right)\left[\int_{\mathbf{Y}} W(\tilde{y}) Q\left(d \tilde{y} \mid y, a^{g}\right)-W(y)\right]\right\} \\
& T(x) \triangleq e^{-\alpha \tau} \inf _{a^{i} \in \mathbf{A}^{i}(y)}\left\{C^{i}\left(y, a^{i}\right)+\int_{\mathbf{Y}} W(\tilde{y}) Q\left(d \tilde{y} \mid y, a^{2}\right)\right\}
\end{aligned}
$$

Since the functions $C^{g}$ and $W$ are bounded and function given by Equation (23) is measurable along the flow $\bar{\phi}$ in $\overline{\mathbf{Y}}$, the integral $\int_{0}^{\bar{t}(x)} C(\phi(x, u)) d u$ is well defined and finite for all $x \in \overline{\mathbf{X}}$, and

$$
\varliminf_{r \rightarrow \infty} V(\phi(x, r))=\varliminf_{r \rightarrow \infty} e^{-\alpha(\tau+r)} W(\bar{\phi}(y, r))=0 ; \quad \varlimsup_{r \rightarrow \infty} V(\phi(x, r))=0
$$

for all $x=(y, \tau) \in \overline{\mathbf{X}}$ with $\bar{t}(x)=\infty$.

Now one can apply Theorem 1. Suppose that a function $V$, having the form as in Equation (24), is a solution to Equation (25), the infimum (with respect to $\theta$ ) is attained on $\Theta(x) \neq \emptyset$ and the set $\Theta(x)$ contains its minimum. Then the Statement 2 of Theorem 1 is valid. And vice versa. We substitute expressions for the functions $C$ and $T$ given in Equation (26) and, after some trivial rearrangements, obtain the following proposition. Condition 1 is required only in order to define the flow in the reverse time and, consequently, to simplify the expression for $\underline{\mathcal{F}}_{-}^{V}(x)$ and the Assertion (i).

Proposition 1. Suppose the flow $\bar{\phi}$ satisfies Condition 1, i.e., for each point $y \in \overline{\mathbf{Y}}$, if $y=$ $\bar{\phi}\left(\tilde{y}_{1}, s_{1}\right)=\bar{\phi}\left(\tilde{y}_{2}, s_{2}\right)$ with $\tilde{y}_{1}, \tilde{y}_{2} \in \overline{\mathbf{Y}}$, then either $\tilde{y}_{1}=\bar{\phi}\left(\tilde{y}_{2}, s_{2}-s_{1}\right)$, or $\tilde{y}_{2}=\bar{\phi}\left(\tilde{y}_{1}, s_{1}-s_{2}\right)$.

Assume that the function $C^{g}$ is bounded and $W$ is a certain bounded function on $\overline{\mathbf{Y}}$ such that the function defined in Equation (23) is measurable along the flow $\bar{\phi}$ in $\overline{\mathbf{Y}}$. Then the following statements are equivalent.

1. The function $W$ satisfies Equation (22) and the following assertions hold true:

- For each $y \in \overline{\mathbf{Y}}$, the infimum in Equation (22) (with respect to $\theta$ ) is attained on $\Theta(y) \neq \emptyset$, and the set $\Theta(y)$ contains its minimum denoted as $\theta^{*}(y) \in \Theta(y)$.

- The function $W$ is measurable along the flow.

2. The function $W$ is such that, for each $y \in \overline{\mathbf{Y}}$, the following quasi-variational inequalities hold 
true

$$
\text { either (a) }\left\{\begin{array}{l}
\lim _{t \rightarrow 0+} \frac{1}{t}\left[e^{-\alpha t} W(\bar{\phi}(y, t))-W(y)+\int_{0}^{t} e^{-\alpha u} \inf _{a^{g} \in \mathbf{A}^{g}(\bar{\phi}(y, u))}\left\{C^{g}\left(\bar{\phi}(y, u), a^{g}\right)\right.\right. \\
\left.\left.+\lambda\left(\bar{\phi}(y, u), a^{g}\right)\left[\int_{\mathbf{Y}} W(\tilde{y}) Q\left(d \tilde{y} \mid \bar{\phi}(y, u), a^{g}\right)-W(\bar{\phi}(y, u))\right]\right\} d u\right]=0 \\
\quad \text { Here necessarily } \bar{t}(y)>0 .) \\
\quad \text { and, in case } y \in \mathbf{Y}^{\text {stop }}, \\
\inf _{a^{i} \in \mathbf{A}^{i}(y)}\left\{C^{i}\left(y, a^{i}\right)+\int_{\mathbf{Y}} W(\tilde{y}) Q\left(d \tilde{y} \mid y, a^{i}\right)\right\}-W(y)>0
\end{array}\right.
$$

or,

$$
\text { if the Assertion (a) fails to hold, then } y \in \mathbf{Y}^{\text {stop }} \text { and }
$$

$$
\left\{\begin{array}{l}
\lim _{t \rightarrow 0-} \frac{1}{t}\left[e^{-\alpha t} W(\bar{\phi}(y, t))-W(y)+\int_{0}^{t} e^{-\alpha u} \inf _{a^{g} \in \mathbf{A}^{g}(\bar{\phi}(y, u))}\left\{C^{g}\left(\bar{\phi}(y, u), a^{g}\right)\right.\right. \\
+\lambda\left(\bar{\phi}(y, u), a^{g}\right)\left[\int_{\mathbf{Y}} W(\tilde{y}) Q\left(d \tilde{y} \mid \bar{\phi}(y, u), a^{g}\right)-W(\bar{\phi}(y, u))\right\} d u \geq 0 \\
\text { (Omit this requirement if y is singular in } \overline{\mathbf{Y}} .) \\
\inf _{a^{i} \in \mathbf{A}^{i}(y)}\left\{C^{i}\left(y, a^{i}\right)+\int_{\mathbf{Y}} W(\tilde{y}) Q\left(d \tilde{y} \mid y, a^{i}\right)\right\}-W(y)=0
\end{array}\right.
$$

and, additionally, the following assertions hold true:

(i) $\quad-$ If $\bar{t}(y)>0$ then $\lim _{t \rightarrow 0+} W(\bar{\phi}(y, t)) \geq W(y)$.

- If $y$ is nonsingular in $\overline{\mathbf{Y}}$ then $\varlimsup_{t \rightarrow 0-} W(\bar{\phi}(y, t)) \leq W(y)$.

(ii) If, for some $s>0$,

$$
\bar{\phi}(y, t) \notin \mathcal{L} \triangleq\left\{\bar{y} \in \mathbf{Y}^{\text {stop }}: \inf _{a^{i} \in \mathbf{A}^{i}(\bar{y})}\left\{g^{i}\left(\bar{y}, a^{i}\right)+\int_{\mathbf{Y}} W(\tilde{y}) Q\left(d \tilde{y} \mid \bar{y}, a^{i}\right)\right\}-W(\bar{y})=0\right\}
$$

for all $0 \leq t<s$, then

$$
\lim _{t \rightarrow s-} W(\bar{\phi}(y, t))=W(\bar{\phi}(y, s)) .
$$

(iii) - The set

$$
\{t \in[0, \bar{t}(y)] \cap \mathbb{R}: \bar{\phi}(y, t) \in \mathcal{L}\}
$$

is not empty if $\bar{t}(y)<\infty$. If it is empty (and necessarily $\bar{t}(y)=\infty$ ), then we put $t^{*}(y) \triangleq \infty$. In case it is not empty, it contains its minimum denoted as $t^{*}(y)$.

In any case, $t^{*}(y) \in \mathbb{T}_{y} \cap[0, \bar{t}(y)]$.

For all $t \in\left[0, t^{*}(y)\right]$, equality $t^{*}(\bar{\phi}(y, t))=t^{*}(y)-t$ is valid and

$$
\begin{aligned}
& e^{-\alpha t} W(\bar{\phi}(y, t))-W(y)+\int_{0}^{t} e^{-\alpha u} \inf _{a^{g} \in \mathbf{A}^{g}(\bar{\phi}(y, u))}\left\{C^{g}\left(\bar{\phi}(y, u), a^{g}\right)\right. \\
& \left.+\lambda\left(\bar{\phi}(y, u), a^{g}\right)\left[\int_{\mathbf{Y}} W(\tilde{y}) Q\left(d \tilde{y} \mid \bar{\phi}(y, u), a^{g}\right)-W(\bar{\phi}(y, u))\right]\right\} d u=0 .
\end{aligned}
$$

The functions $\theta^{*}$ and $t^{*}$ in the Statements 1 and 2 above coincide.

If function given by Equation (23) is continuous along the flow $\bar{\phi}$ (in the sense of Condition 2), and the function $W$ is differentiable along the flow $\bar{\phi}$, then, as explained at the end of Section 2, the first expressions in the Items (a) and (b) take the more familiar form

$$
-\alpha W(y)+W^{\prime}(y)+\inf _{a^{g} \in \mathbf{A}^{g}(y)}\left\{C^{g}\left(y, a^{g}\right)+\lambda\left(y, a^{g}\right)\left[\int_{\mathbf{Y}} W(\tilde{y}) Q\left(d \tilde{y} \mid y, a^{g}\right)-W(y)\right]\right\}=0
$$


and

$$
-\alpha W(y)+W^{\prime}(y)+\inf _{a^{g} \in \mathbf{A}^{g}(y)}\left\{C^{g}\left(y, a^{g}\right)+\lambda\left(y, a^{g}\right)\left[\int_{\mathbf{Y}} W(\tilde{y}) Q\left(d \tilde{y} \mid y, a^{g}\right)-W(y)\right]\right\} \geq 0
$$

correspondingly.

All the presented results are consistent with the works by Dempster and Ye (1995) and Yushkevich (1989), where versions of the impulsively controlled PDMP were investigated: see Theorem 4 in Dempster and Ye (1995) and Theorem 7.2 in Yushkevich (1989). The equivalence of the integral Bellman equation and the quasi-variational inequalities was proved for an impulsiye control problem of PDMP under compactness-continuity and some other assumptions: see Theorem - 5.1. in Costa and Raymundo (2000).

Obviously, if $\lambda(y, a) \equiv 0$, then we obtain a version of the model presented in Subsection 3.1.

\subsection{Impulse control of CTMDP}

This is a special case of the model considered in Subsection 3.2, when there is no flow, that is, when $\bar{\phi}(y, t) \equiv y$. Namely, let $\mathbf{Y}=\overline{\mathbf{Y}}$ be a Borel space,

$$
\bar{t}(y) \equiv \infty, \quad \mathbb{T}_{y}= \begin{cases}{[0, \infty],} & \text { if } \mathbf{A}^{i}(y) \neq \emptyset ; \\ \{\infty\} & \text { otherwise }\end{cases}
$$

and all the other elements $\mathbf{A}, \mathbf{K}^{i}, \mathbf{K}^{g}, \lambda$ etc be as described Subsection 3.2. Clearly, Condition 1 is satisfied and there are no singular points in $\overline{\mathbf{Y}}$. We obtain the impulsively controlled CTMDP studied in Dufour and Piunovskiy (2015).

The Bellman equation (in the differential form) obtained in Dufour and Piunovskiy (2015) looks as follows:

$$
\begin{aligned}
\min & \left\{\inf _{a^{g} \in \mathbf{A}^{g}(y)}\left\{-\alpha w(y)+C^{g}\left(y, a^{g}\right)+\lambda\left(y, a^{g}\right)\left[\int_{\mathbf{Y}} W(\tilde{y}) Q\left(d \tilde{y} \mid y, a^{g}\right)-W(y)\right]\right\} ;\right. \\
& \left.\inf _{a^{i} \in \mathbf{A}^{i}(y)}\left\{C^{i}\left(y, a^{i}\right) \leftarrow \int_{\mathbf{Y}} W(\tilde{y}) Q\left(d \tilde{y} \mid y, a^{i}\right)-W(y)\right\}\right\}=0 .
\end{aligned}
$$

(Recall that $\inf \{\emptyset\}=\infty$. One can recognize here the Statement 2 of Proposition 1: the Assertions (i) and (ii) are trivially valid and

$t^{*}(y)= \begin{cases}0, & \text { if } y \in \mathcal{L}=\left\{\bar{y} \in \mathbf{Y}^{\text {stop }}: \inf _{a^{i} \in \mathbf{A}^{i}(\bar{y})}\left\{C^{i}\left(\bar{y}, a^{i}\right)+\int_{\mathbf{Y}} W(\tilde{y}) Q\left(d \tilde{y} \mid \bar{y}, a^{i}\right)\right\}-W(\bar{y})=0\right\} ; \\ \infty & \text { otherwise. }\end{cases}$

Suppose a bounded function $W$ satisfies Equation (27): sufficient conditions for the existence of $W$ are in Propositions 4.2 and 4.3 of Dufour and Piunovskiy (2015). The very last part of the Assertion (iii), Proposition 1, is valid automatically according to Remark 1(b). Now one can use Proposition 1: the function $W$ satisfies the Bellman Equation (22):

$$
\begin{aligned}
W(y)= & \inf _{\theta \in \mathbb{T}_{y}}\left\{\int_{0}^{\theta} e^{-\alpha u} \inf _{a^{g} \in \mathbf{A}^{g}(y)}\left\{C^{g}\left(y, a^{g}\right)+\lambda\left(y, a^{g}\right)\left[\int_{\mathbf{Y}} W(\tilde{y}) Q\left(d \tilde{y} \mid y, a^{g}\right)-W(y)\right]\right\} d u\right. \\
& \left.+e^{-\alpha \theta} \inf _{a^{i} \in \mathbf{A}^{i}(y)}\left\{C^{i}\left(y, a^{i}\right)+\int_{\mathbf{Y}} W(\tilde{y}) Q\left(d \tilde{y} \mid y, a^{i}\right)\right\}\right\}, \quad y \in \mathbf{Y},
\end{aligned}
$$

and the infimum with respect to $\theta$ is provided by $\theta^{*}(y)=t^{*}(y)$. The established shape of the function $\theta^{*}$ means that the impulse must be applied either immediately as soon as the state $y \in \mathcal{L}$ is reached, or never (if $y \notin \mathcal{L}$, one should wait till the next 'natural' jump governed by the jump rate $\lambda$ ). This property of the optimal strategy was established in Dufour and Piunovskiy (2015), and simultaneous sequences of impulses were there allowed. 


\subsection{Impulse and gradual control of dynamical systems}

Consider the following optimal control problem.

- $\mathbf{Y}=\mathbb{R}^{d}$ is the state space;

- $\mathbf{A}^{g}$ is the Borel space of gradual actions admissible at all the states $y \in \mathbf{Y}$.

Between the impulses, the system evolves according to the differential equation

$$
\dot{y}=f(y, \varphi(y)),
$$

where $f: \mathbf{Y} \times \mathbf{A}^{g} \rightarrow \mathbf{Y}$ is a measurable function and $\varphi: \quad \mathbf{Y} \rightarrow \mathbf{A}^{g}$ is an arbitrary measurable mapping (feedback control). We assume that, for each such $\varphi$, differential Equation (28) satisfies the standard conditions leading to the existing of the unique function $y^{\varphi}(t), t \geq 0$ satisfying an (arbitrarily) fixed initial condition $y(0)=y_{0} \in \mathbf{Y}$. As a result, we obtain the flow $\phi^{\varphi}: \phi^{\varphi}\left(y_{0}, t\right)=$ $y^{\varphi}(t)$.

Further, suppose

- $\mathbf{A}^{i}$ is the Borel space of impulsive actions admissible at all the states $y \in \mathbf{Y}$, which can be applied at any time moment;

- $l\left(y, a^{i}\right)$ is the new state immediately after the impulse $a^{i}$ applied at the state $y$;

- $C^{g}\left(y, a^{g}\right), y \in \mathbf{Y}, a^{g} \in \mathbf{A}^{g}$ is the bounded measurable (gradual) cost rate;

- $C^{i}\left(y, a^{i}\right) \geq \delta>0$ is the cost associated with the impulse $a^{i} \in \mathbf{A}^{i}$ applied at the state $y \in \mathbf{Y}$.

We consider the discounted optimal control problem with the discount factor $\alpha>0$ :

$$
\varlimsup_{T \rightarrow \infty}\left[\int_{0}^{T} e^{-\alpha t} C^{g}\left(y^{\varphi}(t), \varphi\left(y^{\varphi}(t)\right)\right) d t+\sum_{T_{j} \in[0, T]} e^{-\alpha T_{j}} C^{i}\left(y^{\varphi}\left(T_{j}\right), a_{j}^{i}\right)\right] \rightarrow \inf _{\pi} .
$$

Here the initial condition $y(0)=y_{0}$ is fixed, $0 \leq T_{0}<T_{1}<\ldots$ are the time moments when the impulses $a_{j}^{i}$ are applied and $\pi=\left\{\varphi,\left(T_{j}, a_{j}^{i}\right)_{j=0}^{\infty}\right\}$ is a control strategy. On each time interval $\left(T_{j}, T_{j+1}\right]$, the trajectory $y^{\varphi}(\cdot)$ starts from the state $l\left(y^{\varphi}\left(T_{j}\right), a_{j}^{i}\right)$ and evolves according to the differential Equation (28). Note that $T_{j}$ can equal $\infty$ (meaning, no impulses in the future). Simultaneous infinite sequence of impulses is not optimal because of the inequality $C^{i}\left(y, a^{i}\right) \geq \delta>0$, and a simultaneous finite sequence of impulses can be denoted as the "super-impulse", so that assumption $T_{j}<T_{j+1}$ for $T_{j}<\infty$ is not restricting. Such a model was considered in Avrachenkov et al. (2015).

The dynamic programming approach in Avrachenkov et al. (2015) was based on the differential Bellman equation

$$
\begin{aligned}
\min & \left\{\inf _{a^{g} \in \mathbf{A}^{g}}\left\{C^{g}\left(y, a^{g}\right)-\alpha W(y)+\left\langle\frac{\partial W(y)}{\partial y}, f\left(y, a^{g}\right)\right\rangle\right\}\right. \\
& \left.\inf _{a^{i} \in \mathbf{A}^{i}}\left\{C^{i}\left(y, a^{i}\right)+W\left(l\left(y, a^{i}\right)\right)-W(y)\right\}\right\}=0, \quad y \in \mathbf{Y} .
\end{aligned}
$$

We leave aside the conditions when this equation has a solution coincident with the Bellman function (the minimal discounted cost when starting from the state $y \in \mathbf{Y}$ ) and also the properties of the function $W$ and of the infima with respect to $a^{g}$ and $a^{i}$ in Equation (30). The interested reader can find a meaningful example of the successful solving Equation (30) and constructing the optimal control strategy in Avrachenkov et al. (2015). We only intend to illustrate how one can apply Theorem 1 in this situation. Note, the flow in $\mathbf{Y}$ is not fixed so far.

Assumption 1. (a) There is a bounded function $W$ on $\mathbf{Y}$, everywhere differentiable and satisfying Equation (30).

(b) There is a measurable mapping $\varphi^{*}(y): \mathbf{Y} \rightarrow \mathbf{A}^{g}$ providing the first infimum in Equation (30). 
The corresponding flow in $\mathbf{Y}$ is denoted as $\phi^{*} \triangleq \phi^{\varphi^{*}}$ and satisfies Condition 1 (where $\overline{\mathbf{X}}=\mathbf{Y}$ ).

(c) The function $C^{g}\left(y, \varphi^{*}(y)\right)$ is continuous along the flow $\phi^{*}$ (in the sense of Condition 2).

(d) For each $y \in \mathbf{Y}$ the infimum (which may equal $\infty$ )

$$
\inf \left\{t \geq 0: \phi^{*}(y, t) \in \mathcal{L} \triangleq\left\{\bar{y}: \inf _{a^{i} \in \mathbf{A}^{i}}\left\{C^{i}\left(\bar{y}, a^{i}\right)+W\left(l\left(\bar{y}, a^{i}\right)\right)\right\}-W(\bar{y})=0\right\}\right\} \triangleq t^{*}(y)
$$

is attained.

Below, we assume that Assumption 1 is fulfilled. It was proved in Avrachenkov et al. (2015) that, under Assumption 1, if there is a measurable mapping $\psi^{*}(y): \mathbf{Y} \rightarrow \mathbf{A}^{i}$ proyiding the second infimum in Equation (30), then the following control strategy is optimal in the problem given by Equation (29): $\varphi=\varphi^{*}$, and the impulse $\psi^{*}(y)$ should be applied immediately as soon as $y^{\varphi^{*}}(t) \in \mathcal{L}$. This informal description can be reformulated as the strategy $\pi^{*}=\left\{\varphi^{*},\left(T_{j}^{*}, a_{j}^{i *}\right)_{j=0}^{\infty}\right\}$.

Similarly to Subsection 3.2 , we extend the state space to $\mathbf{Z} \triangleq \mathbb{R}^{d+1}$ adding the time component, and put

$$
\overline{\mathbf{X}} \triangleq \mathbf{Z} ; \quad \mathbb{T}_{x} \triangleq[0, \infty] ; \quad \bar{t}(x) \equiv \infty ; \quad \mathbf{X}^{\text {stop }} \triangleq \mathbf{Z}
$$

The generic elements of $\mathbf{Z}$ are denoted as $x=(y, \tau)$ and $\phi(x, t) \triangleq\left(\phi^{*}(y, t), \tau+t\right)$ is the flow in $\mathbf{Z}$ possessing the semigroup property and satisfying Condition 1 by Assumption 1(b). Finally, having the function $W$ and the mapping $\varphi^{*}$, we define

$$
\begin{aligned}
& V(x) \triangleq e^{-\alpha \tau} W(y) \\
& C(x) \triangleq e^{-\alpha \tau} C^{g}\left(y, \varphi^{*}(y)\right) ; \\
& T(x) \triangleq e^{-\alpha \tau} \inf _{a^{i} \in \mathbf{A}^{i}}\left\{q^{i}\left(y, a^{i}\right)+W\left(l\left(y, a^{i}\right)\right)\right\}
\end{aligned}
$$

for all $x=(y, \tau) \in \overline{\mathbf{X}}$.

Now the differential Bellman Equation (30) is fulfilled if and only if the function $V$ satisfies Equation (17), where

$$
V^{\prime}(x)=e^{-\alpha \tau}\left[-\alpha W(y)+\left\langle\frac{\partial W}{\partial y}, f\left(y, \varphi^{*}(y)\right)\right\rangle\right]
$$

is the derivative of $V$ along the flow $\phi$ (the right derivative if $x$ is singular in $\overline{\mathbf{X}}$ ).

We can apply Theorem 1 to the function $V$ and the flow $\phi$. Requirements $\lim _{r \rightarrow \infty} V(\phi(x, r))=0$ and $\left|\int_{0}^{\infty} C(\phi(x, u)) d u\right|<\infty$ are fulfilled because the functions $W$ and $C^{g}$ are bounded. The Assertions (i) and (ii) in Theorem 1 hold true because of Assumption 1(a), and the Assertion (iii) is valid because of Assumption 1(d). The last part of the Assertion (iii) is not needed by Remark $1(\mathrm{~b})$.

According to Theorem 1, the Statement 1 holds for the function $V$. We substitute the expressions for the functions $C$ and $T$ and conclude that the function $W$ satisfies the following integral Bellman equation

$$
\begin{aligned}
W(y)= & \inf _{\theta \in[0, \infty]}\left\{\int_{0}^{\theta} e^{-\alpha u} C^{g}\left(\phi^{*}(y, u), \varphi^{*}\left(\phi^{*}(y, u)\right)\right) d u\right. \\
& \left.+I\{\theta<\infty\} e^{-\alpha \theta} \inf _{a^{i} \in \mathbf{A}^{i}}\left\{C^{i}\left(\phi^{*}(y, \theta), a^{i}\right)+W\left(l\left(\phi^{*}(y, \theta), a^{i}\right)\right)\right\}\right\}, y \in \mathbf{Y}
\end{aligned}
$$

and, for each $y \in \mathbf{Y}$, the infimum with respect to $\theta$ is attained on $\Theta(y) \neq \emptyset$, and the set $\Theta(y)$ contains its minimum denoted as $\theta^{*}(y) \in \Theta(y)$. Moreover, $\theta^{*}(y) \equiv t^{*}(y)$.

One can rewrite Equation (32) in the intuitively clearer way. 
Let $\theta \in[0, \infty]$ and $y(0)=y_{0} \in \mathbf{Y}$ be arbitrarily fixed. For any gradual feedback control $\varphi$,

$$
\begin{aligned}
& \int_{0}^{\theta} e^{-\alpha u} C^{g}\left(y^{\varphi}(u), \varphi\left(y^{\varphi}(u)\right)\right) d u+I\{\theta<\infty\} e^{-\alpha \theta} W\left(y^{\varphi}(\theta)\right) \\
= & \int_{0}^{\theta} e^{-\alpha u}\left[C^{g}\left(y^{\varphi}(u), \varphi\left(y^{\varphi}(u)\right)\right)-\alpha W\left(y^{\varphi}(u)\right)+\left\langle\frac{\partial W\left(y^{\varphi}(u)\right)}{\partial y}, f\left(y^{\varphi}(u), \varphi\left(y^{\varphi}(u)\right)\right)\right\rangle\right] d u \\
& -I\{\theta<\infty\} e^{-\alpha \theta} W\left(y^{\varphi}(\theta)\right)+W\left(y_{0}\right)+I\{\theta<\infty\} e^{-\alpha \theta} W\left(y^{\varphi}(\theta)\right) \\
\geq & W\left(y_{0}\right)
\end{aligned}
$$

according to Equation (30).

Remark 2. The feedback $\varphi^{*}$ provides the equality in Equation (33) if $\theta \leq \theta^{*}\left(y_{0}\right)$. Indeed, for all $u<\theta^{*}\left(y_{0}\right)=t^{*}\left(y_{0}\right)$, we have $y^{\varphi^{*}}(u)=\phi^{*}\left(y_{0}, u\right) \notin \mathcal{L}$, i.e.,

$$
\inf _{a^{i} \in \mathbf{A}^{i}}\left\{C^{i}\left(y^{\varphi^{*}}(u), a^{i}\right)+W\left(l\left(y^{\varphi^{*}}(u), a^{i}\right)\right)\right\}-W\left(y^{\varphi^{*}}(u)>0\right.
$$

so that

$$
\begin{aligned}
& \inf _{a^{g} \in \mathbf{A}^{g}}\left\{C^{g}\left(y^{\varphi^{*}}(u), a^{g}\right)-\alpha W\left(y^{\varphi^{*}}(u)\right)+\left\langle\frac{\partial W\left(y^{\varphi^{*}}(u)\right)}{\partial y}, f\left(y^{\varphi^{*}}(u), a^{g}\right)\right\rangle\right\} \\
= & C^{g}\left(y^{\varphi^{*}}(u), \varphi^{*}\left(y^{\varphi^{*}}(u)\right)\right)-\alpha W\left(y^{\varphi^{*}}(u)\right)+\left\langle\frac{\partial W\left(y \varphi^{*}(u)\right)}{\partial y}, f\left(y^{\varphi^{*}}(u), \varphi^{*}\left(y^{\varphi^{*}}(u)\right)\right)\right\rangle \\
= & 0 .
\end{aligned}
$$

Hence, if $\theta \leq \theta^{*}\left(y_{0}\right)$, then $\varphi^{*}$ is optimal in the problem

$$
\int_{0}^{\theta} e^{-\alpha u} C^{g}\left(y^{\varphi}(u), \varphi\left(y^{\varphi}(u)\right)\right) d u+I\{\theta<\infty\} e^{-\alpha \theta} W\left(y^{\varphi}(\theta)\right) \rightarrow \inf _{\varphi}
$$

and the minimal value equals $W\left(y_{0}\right)$. This is the standard reasoning for the dynamic programming approach: see Ch.IV of Fleming and Rishel (1975). Note that the optimal gradual feedback $\varphi^{*}$ does not depend on the initial state $y_{0}$.

Now it is clear that for each $y_{0} \in \mathbf{Y}$,

$$
\begin{aligned}
& W\left(y_{0}\right) \\
& \geq \inf _{\theta \in[0, \infty]} \inf _{\varphi}\left\{\int_{0}^{\theta} e^{-\alpha u} C^{g}\left(y^{\varphi}(u), \varphi\left(y^{\varphi}(u)\right)\right) d u\right. \\
& \geq \inf _{\theta \in[0, \infty]} \inf _{\varphi}\left\{\int_{0}^{\theta} e^{-\alpha u} C^{g}\left(y^{\varphi}(u), \varphi\left(y^{\varphi}(u)\right)\right) d u+I\{\theta<\infty\} e^{-\alpha \theta} W\left(y^{\varphi}(\theta)\right)\right\} \\
& \inf _{\theta \in[0, \infty]} W\left(y_{0}\right)=W\left(y_{0}\right) .
\end{aligned}
$$

Here the first inequality is by Equation (32), the second inequality is by Equation (30), and the third inequality is by Equation (33).

We conclude that the function $W$ satisfies also the following version of the integral Bellman equation:

$$
\begin{aligned}
W\left(y_{0}\right)= & \inf _{\theta \in[0, \infty]} \inf _{\varphi}\left\{\int_{0}^{\theta} e^{-\alpha u} C^{g}\left(y^{\varphi}(u), \varphi\left(y^{\varphi}(u)\right)\right) d u\right. \\
& \left.+I\{\theta<\infty\} e^{-\alpha \theta} \inf _{a^{i} \in \mathbf{A}^{i}}\left\{C^{i}\left(y^{\varphi}(\theta), a^{i}\right)+W\left(l\left(y^{\varphi}(\theta), a^{i}\right)\right)\right\}\right\}
\end{aligned}
$$


for all $y_{0} \in \mathbf{Y}$, and this equation clearly appears from the intuitive dynamic programming reasoning. If $W(y)$ is the minimal discounted cost when starting from the state $y \in \mathbf{Y}$, then, firstly, under an arbitrarily fixed $\theta \in[0, \infty]$, the interval till the next one impulse, one has to choose such a gradual feedback which minimises the total discounted cost on the interval $[0, \theta]$, including the minimal expected future cost associated with the impulse $a^{i}$. One accepts that, after the impulse, the future (minimal) cost is $W(\tilde{y})$ if the state after the impulse is $\tilde{y}$. Secondly, after that, one has to optimize the interval $\theta$.

Note also that

$$
\begin{aligned}
W\left(y_{0}\right)= & \int_{0}^{\theta^{*}\left(y_{0}\right)} e^{-\alpha u} C^{g}\left(y^{\varphi^{*}}(u), \varphi^{*}\left(y^{\varphi^{*}}(u)\right)\right) d u \\
& +I\left\{\theta^{*}\left(y_{0}\right)<\infty\right\} e^{-\alpha \theta^{*}\left(y_{0}\right)} \inf _{a^{i} \in \mathbf{A}^{i}}\left\{C^{i}\left(y^{\varphi^{*}}\left(\theta^{*}\left(y_{0}\right)\right), a^{i}\right)+W\left(l\left(y^{\varphi^{*}}\left(\theta^{*}\left(y_{0}\right)\right), a^{i}\right)\right)\right\}
\end{aligned}
$$

by Equation (32) and the optimal gradual feedback $\varphi^{*}$ does not depend on $y_{0}$ according to Remark 2 .

Equation (34) is convenient for the numerical solving by successive approximations. If, e.g., $C^{g}, C^{i} \geq 0$ then the sequence of functions

$$
\begin{aligned}
W_{0}\left(y_{0}\right)= & 0 ; \\
W_{j+1}\left(y_{0}\right)= & \inf _{\theta \in[0, \infty]} \inf _{\varphi}\left\{\int_{0}^{\theta} e^{-\alpha u} C^{g}\left(y^{\varphi}(u), \varphi\left(y^{\varphi}(u)\right)\right) d u\right. \\
& \left.+I\{\theta<\infty\} e^{-\alpha \theta} \inf _{a^{i} \in \mathbf{A}^{i}}\left\{C^{i}\left(y^{\varphi}(\theta), a^{i}\right)+W_{j}\left(l\left(y^{\varphi}(\theta), a^{i}\right)\right)\right\}\right\}
\end{aligned}
$$

increases. Under appropriate conditions, one can show that the limiting function $W(y)=\lim _{j \rightarrow \infty} W_{j}(y)$ satisfies Equation (34) and coincides with the Bellman function (the minimal discounted cost when starting from the state $y \in \mathbf{Y})$. One can find similar rigorous reasoning in Dufour and Piunovskiy (2015) and in Dufour et al. (2016).

In the inverse direction, suppose a bounded function $W$ satisfies the Bellman Equation (34) and all the infima are attained. Moreover, assume that, the infimum with respect to $\theta$ is attained on $\Theta\left(y_{0}\right) \neq \emptyset$ and $\theta^{*}\left(y_{0}\right) \triangleq \inf \Theta\left(y_{0}\right) \in \Theta\left(y_{0}\right)$, and there is a gradual (measurable) feedback $\varphi^{*}$ providing the infimum with respect to $\varphi$ under $\theta^{*}\left(y_{0}\right)$ simultaneously for all $y_{0} \in \mathbf{Y}$. The latter assumption is very often satisfied: see Remark 2. Then the function $V(x)=e^{-\alpha \tau} W(y)$, where $x=(y, \mathcal{T})$, satisfies the integral Bellman Equation (2) for the flow $\phi^{*}$ in the extended state space $\overline{\mathbf{X}}=\mathbf{Z}=\mathbb{R}^{d+1}$, defined as $\phi^{*}((y, \tau), t) \triangleq\left(\phi^{\varphi^{*}}(y, t), \tau+t\right)$. The functions $C(x)$ and $T(x)$ are as introduced above in Equation (31). We see that the Statement 1 of Theorem 1 is valid. (The very last assertion can be ignored according to Remark 1(a).) With some abuse of notation, $\theta^{*}(x)=\theta^{*}(y)$ for all $x=(y, \tau) \in \overline{\mathbf{X}}$. Thus, the Statement 2 is valid. As a result, we see that the differential Bellman equation, instead of Equation (30), can be formulated in the more general form of Equation (3), where the function $W$ may be not differentiable and Assumption 1 can fail to hold.

There are other ways how one can apply Theorem 1 to Equation (30). Suppose, instead of the total Assumption 1, only Assumption 1(a) is fulfilled.

Like previously, we extend the state space to $\mathbf{Z} \triangleq \mathbb{R}^{d+1}$ by adding the time component and put

$$
\phi(x, t) \triangleq(y, \tau+t)
$$

for $x=(y, \tau) \in \mathbf{Z}$. Further,

$$
\begin{gathered}
\overline{\mathbf{X}} \triangleq \mathbf{Z} ; \quad \mathbb{T}_{x} \triangleq[0, \infty] ; \quad \bar{t}(x) \equiv \infty ; \quad \mathbf{X}^{\text {stop }} \triangleq \mathbf{Z} ; \\
c(y) \triangleq \inf _{a^{g} \in \mathbf{A}^{g}}\left\{C^{g}\left(y, a^{g}\right)-\alpha W(y)+\left\langle\frac{\partial W(y)}{\partial y}, f\left(y, a^{g}\right)\right\rangle\right\}
\end{gathered}
$$


and

$$
\begin{aligned}
C(x) & \triangleq e^{-\alpha \tau} c(y) \\
T(x) & \triangleq e^{-\alpha \tau}\left[\inf _{a^{i} \in \mathbf{A}^{i}}\left\{C^{i}\left(y, a^{i}\right)+W\left(l\left(y, a^{i}\right)\right)\right\}-W(y)\right] .
\end{aligned}
$$

Now Equation (30) takes the form

$$
\min \{C(x), T(x)\}=0,
$$

and function $V(x) \equiv 0$ satisfies the Statement 2 of Theorem 1, leading to Equation (2): for $x=(y, \tau) \in \mathbf{Z}$,

$$
\begin{aligned}
0= & e^{-\alpha \tau} \inf _{\theta \in[0, \infty]}\left\{\int_{0}^{\theta} e^{-\alpha u} c(y) d u+e^{-\alpha \theta} I\{\theta<\infty\} \inf _{a^{i} \in \mathbf{A}^{i}}\left\{C^{i}\left(y, a^{i}\right)+W\left(l\left(y, a^{i}\right)\right)-W(y)\right\}\right\} \\
0 & \inf _{\theta \in[0, \infty]}\left\{\frac{1-e^{-\alpha \theta}}{\alpha} \inf _{a^{g} \in \mathbf{A}^{g}}\left\{C^{g}\left(y, a^{g}\right)-\alpha W(y)+\left\langle\frac{\partial W(y)}{\partial y}, f\left(y, a^{g}\right)\right)\right\}\right. \\
& \left.+e^{-\alpha \theta} \inf _{a^{i} \in \mathbf{A}^{i}}\left\{C^{i}\left(y, a^{i}\right)+W\left(l\left(y, a^{i}\right)\right)-W(y)\right\}\right\} .
\end{aligned}
$$

Now

$$
\theta^{*}(x)=t^{*}(x)= \begin{cases}0, & \text { if } \inf _{a^{i} \in \mathbf{A}^{i}}\left\{C^{i}\left(\widehat{y, a^{i}}\right)+W\left(l\left(y, a^{i}\right)\right)-W(y)\right\}=0 \\ \infty & \text { otherwise. }\end{cases}
$$

Actually, we obtained nothing new: Equation (35) is satisfied if and only if Equation (30) is satisfied.

\section{Conclusion}

Theorem 1 is purely analytic, showing the equivalence of two statements or, roughly speaking, of two Equations (2) and (3). In Section 3, we illustrate how one can use it when investigating several different optimal impulse control problems.

Note also that Subsections 3.2 and 3.4 can be considered as the addendum to Dufour and Piunovskiy (2015); Dufour et al. (2016), and to Avrachenkov et al. (2015).

\section{Acknowledgement}

This research was supported by the Royal Society International Exchanges award IE160503.

\section{Appendix}

Lemma 1. Let $t \in[0, \infty]$. If a real-valued function $h$ is defined on $[0, t] \cap \mathbb{R}$ and for all $s \in(0, t)$

$$
\text { either } \quad \underline{h}_{-}^{\prime}(s) \triangleq \varliminf_{t \rightarrow 0^{+}} \frac{h(s)-h(s-t)}{t} \geq 0 \quad \text { or } \quad \underline{h}_{+}^{\prime}(s) \triangleq \varliminf_{t \rightarrow 0_{+}} \frac{h(s+t)-h(s)}{t} \geq 0,
$$

and, additionally, $h$ is right lower semicontinuous on $[0, t)$ and left upper semicontinuous on $(0, t] \cap$ $\mathbb{R}$, then $h$ is monotone nondecreasing.

Lemma 2. Let $t \in[0, \infty]$. If a real-valued function $h$ is defined on $[0, t] \cap \mathbb{R}$ and is left continuous on $(0, t] \cap \mathbb{R}$, and $h_{+}^{\prime}(s)=0$ for $s \in[0, t)$ then $h$ is constant.

One can find the proofs in Piunovskiy et al. (2019a). 


\section{References}

[1] Abakuks, A. (1973). An optimal isolation policy for an epidemic. J. Appl. Probab., 10, 247262 .

[2] Avrachenkov, K., Habachi, O., Piunovskiy, A., \& Zhang, Y. (2015). Infinite horizon optimal impulsive control with applications to Internet congestion control. Intern. J. of Control, 88, 703-716.

[3] Bensoussan, A., \& Lions, J.L. (1984). Impulse control and quasivariational inequalities, Montrouge: Gauthier-Villars.

[4] Costa, O.L.V., \& Raymundo, C.A.B. (2000). Impulse and continuous control of piecewise deterministic Markov processes, Stochastics Stochastics Rep., 70(1-2), 75-107.

[5] de Saporta, B., Dufour, F., \& Geeraert, A. (2017). Optimal strategies for impulsive control of piecewise deterministic Markov processes. Automatica, 77, 219-229.

[6] Dempster, M.A.H., \& Ye, J.J. (1995). Impulse control of piecewise deterministic Markov processes. Ann. Appl. Probab., 5(2), 399-423.

[7] Dufour, F., \& Piunovskiy, A. (2015). Impulsive control for continuous-time Markov decision processes. Adv. Appl. Prob., 47, 106-127.

[8] Dufour, F., Horiguchi, M., \& Piunovskiy, A. (2016). Optimal impulsive control of piecewise deterministic Markov processes. Stochastics, 88(7), 1073-1098.

[9] Fleming, W.H., \& Rishel, R.W. (1975). Deterministic and stochastic optimal control. Berlin: Springer.

[10] Hou, S.H., \& Wong, K.H. (2011). Optimal impulsive control problem with application to human immunodeficiency virus treatment. J. Optim. Theory Appl., 151, 385-401.

[11] Leander, R., Lenhart, S., \& Protopopescu, V. (2015). Optimal control of continuous systems with impulse controls. Optim Control Appl. Meth., 36, 535-549.

[12] Menaldi, J.L., \& Robin, M. (2017). On some impulse control problems with constraint. SIAM J. Control Optim. 55(5), 3204-3225.

[13] Peskir, G. \& Shiryaev, A. (2006). Optimal stopping and free-boundary problems. Basel: Birkhäuser Verlag.

[14] Piunoyskiy, A., Plakhov, A., Torres, D.F.M., \& Zhang, Y. (2019a). Optimal impulse control of dynamical systems. SIAM J. Control Optim., 57(4), 2720-2752.

[15] Piunovskiy, A., Plakhov, A., \& Tumanov, M. (2019b). Optimal impulse control of a SIR epidemic. Optim. Control Appl. Meth. Advance online publication. doi: 10.1002/oca.2552

[16] Wei, J., Yang, H., \& Wang, R. (2011). Classical and impulse control for the optimization of dividend and proportional reinsurance policies with regime switching. J. Optim. Theory Appl., 147, 358-377.

[17] Yushkevich, A.A. (1989). Verification theorems for Markov decision processes with controlled deterministic drift and gradual and impulsive controls. Teor. Probab. Appl., 34(3), 474-496. 